\title{
On asymptotic approximations of first integrals for second order difference equations
}

\author{
M. Rafei · W.T. Van Horssen
}

Received: 13 November 2009 / Accepted: 19 January 2010 / Published online: 5 February 2010

(C) The Author(s) 2010. This article is published with open access at Springerlink.com

\begin{abstract}
In this paper, the concept of invariance factors for second order difference equations to obtain first integrals or invariants will be presented. It will be shown that all invariance factors have to satisfy a functional equation. Van Horssen (J. Indones. Math. Soc. 13:1-15, 2007) developed a perturbation method for a single first order difference equation based on invariance factors. This perturbation method will be reviewed shortly, and will be extended to second order difference equations. Also, in this paper, we will construct approximations of first integrals for second order linear, and weakly nonlinear difference equations.
\end{abstract}

Keywords Invariance vector · First integrals . Functional equation - Difference equation - Van der Pol type of nonlinearity

\section{Introduction}

For scientists and engineers, the analysis of nonlinear dynamical systems is an important field of research since the solutions of these systems can exhibit

M. Rafei $(\bowtie) \cdot$ W.T. Van Horssen

Delft Institute of Applied Mathematics (DIAM), Delft University of Technology, Mekelweg 4, 2628 CD Delft, The Netherlands

e-mail: M.Rafei@tudelft.nl

W.T. Van Horssen

e-mail: W.T.VanHorssen@tudelft.nl counterintuitive and sometimes unexpected behavior. To obtain useful information from these systems, the construction of (approximations of) first integrals by means of computing (approximate) integrating factors can play an important role.

The fundamental concept of how to make a single first order ordinary differential equation (ODE) exact by means of integrating factors was discovered by Euler in the period 1732-1734. Euler showed that for a first order ODE all of integrating factors have to satisfy a single, first order, linear partial differential equation. Finding an integrating factor for a given first order ODE was and still is a difficult and usually impossible task. Euler, however, used special types of integrating factors obtaining (and so solving) classes of first order ODEs. In the latter part of the nineteenth century, Sophus Lie introduced the notion of continuous groups (currently known as Lie groups) to unify and to extend various solution methods for ODEs. Lie also showed that the existence of an integrating factor is equivalent to having a Lie group invariance or symmetry of the given ODE. To find a symmetry for a given (system of) $\mathrm{ODE}(\mathrm{s})$ is in general, however, extremely difficult and usually an impossible task. In Van Horssen's papers [2-4], the fundamental concept of how to make second and higher order ODEs as well as systems of first order ODEs exact by means of integrating factors and integrating vectors has been presented. Like most methods for differential equations there is an analogous method for difference equations (see, for instance, [5, 6]). Recently, first integrals, invariants and Lie group theory 
for ordinary difference equations $(\mathrm{O} \Delta \mathrm{Es})$ obtained a lot of attention in the literature (see, for instance, the list of references in [7]). Also recently, the fundamental concept of invariance factors for $\mathrm{O} \Delta \mathrm{Es}$ to obtain invariants (or first integrals) for $\mathrm{O} \Delta \mathrm{Es}$ has been presented in [7].

It has been shown in [7] that in finding invariants for a system of first order difference equations all invariance factors have to satisfy a functional equation (for more information on functional equations and how to solve some of them we refer the reader to [8-13]). The aim of this paper is to construct asymptotic approximations of first integrals for a system of first order $\mathrm{O} \Delta \mathrm{Es}$. After presenting the concepts, we will explicitly show how invariants for a linear, second order difference equation, and also for a second order weakly nonlinear difference equation (with a Van der Pol type of nonlinearity) can be constructed. To obtain highly accurate approximations of invariants, it also will be proposed to include the method of multiplescales into the perturbation method based on invariance factors. It is interesting to notice that for difference equations a formulation of the multiple-scales methods completely in terms of difference operators has also recently been developed in [14].

The outline of this paper is as follows. In Sect. 2, the concept of invariance factors for a system of first order $\mathrm{O} \Delta$ Es will be given, and in Sect. 3 some of the first results in the development of a perturbation method for a system of $\mathrm{O} \Delta$ Es based on invariance factors will be presented. In Sects. 4 and 5, approximations of first integrals for systems of linear and weakly nonlinear $\mathrm{O} \Delta \mathrm{Es}$, respectively, will be constructed. Finally, in Sect. 6 of this paper, some conclusions will be drawn and some future directions for research will be indicated.

\section{On invariance factors for $\mathrm{O} \Delta \mathrm{Es}$}

In this section, we are going to present an overview of the concept of invariance factors (or vectors) for a system of $k$ first order $\mathrm{O} \Delta \mathrm{Es}$ (where $k$ is fixed and $k \in \mathbb{N}$ ), for the reference concerning this concept, we refer the reader to [1]. Consider

$\underline{x}_{n+1}=\underline{f}\left(\underline{x}_{n}, n\right)$,

for $n=0,1,2, \ldots$, and where $\underline{x}_{n}=\left(x_{1, n}, x_{2, n}, \ldots\right.$, $\left.x_{k, n}\right)^{T}, \underline{f}=\left(f_{1}, f_{2}, \ldots, f_{k}\right)^{T}$ in which the superscript indicates the transpose, and where $f_{i}=f_{i}\left(\underline{x}_{n}, n\right)=$ $f_{i}\left(x_{1, n}, x_{2, n}, \ldots, x_{k, n}, n\right)$ are sufficiently smooth functions (for $i=1,2, \ldots, k$ ). We also assume that an invariant for (1) can be represented by

$$
\begin{aligned}
I\left(\underline{x}_{n+1}, n+1\right) & =I\left(\underline{x}_{n}, n\right)=\text { constant } \\
& \Leftrightarrow \Delta I\left(\underline{x}_{n}, n\right)=0 .
\end{aligned}
$$

We now try to find an invariant for (1). By multiplying each $i$ th equation, in (1), with a factor $\mu_{i}\left(\underline{x}_{n+1}, n+1\right)=\mu_{i}\left(\underline{f}\left(\underline{x}_{n}, n\right), n+1\right)$ for $i=1,2, \ldots$, $k$ and by adding the resulting equations, we obtain

$$
\begin{aligned}
\underline{\mu}\left(\underline{x}_{n+1}, n+1\right) \cdot \underline{x}_{n+1}= & \underline{\mu}\left(\underline{f}\left(\underline{x}_{n}, n\right), n+1\right) \\
& \cdot \underline{f}\left(\underline{x}_{n}, n\right),
\end{aligned}
$$

where $\mu=\left(\mu_{1}, \mu_{2}, \ldots, \mu_{k}\right)^{T}$, and $\mu_{i}=\mu_{i}\left(\underline{x}_{n}, n\right)=$ $\mu_{i}\left(x_{1, n}, x_{2, n}, \ldots, x_{k, n}, n\right)$ for $i=1,2, \ldots, k$. In fact, when $\mu$ is an invariance vector we now obtain an exact difference equation (2). The relationship between $I$ and $\underline{\mu}$ follows from the equivalence of (2) and (3), yielding

$\left\{\begin{array}{l}I\left(\underline{x}_{n+1}, n+1\right)=\mu\left(\underline{x}_{n+1}, n+1\right) \cdot \underline{x}_{n+1}, \\ \left.I\left(\underline{x}_{n}, n\right)=\underline{\mu}\left(\underline{f}^{\left(x_{n}\right.}, n\right), n+1\right) \cdot \underline{f}\left(\underline{x}_{n}, n\right) .\end{array}\right.$

By reducing the index $n+1$ by 1 in the first part of (4) I can be eliminated from (4), and then it follows that all invariance vectors for the system of difference equations (1) have to satisfy the functional equation

$\underline{\mu}\left(\underline{x}_{n}, n\right) \cdot \underline{x}_{n}=\underline{\mu}\left(\underline{f}\left(\underline{x}_{n}, n\right), n+1\right) \cdot \underline{f}\left(\underline{x}_{n}, n\right)$.

When an invariance vector has been determined from the functional equation (5) an invariant for (1) easily follows from (4), yielding

$I\left(\underline{x}_{n}, n\right)=\underline{\mu}\left(\underline{x}_{n}, n\right) \cdot \underline{x}_{n}$.

Finding an invariance vector for a given system of first order difference equations is a difficult and usually impossible task. On the other hand, we can use invariance vectors of some special form, and so we can obtain invariants for special classes of systems of $k$ first order difference equations. Examples of this approach have been given in [7].

\section{A perturbation method based on invariance factors for systems of first order $\mathrm{O} \Delta \mathrm{Es}$}

In this section, some of the first results in the development of a perturbation method for difference equa- 
tion based on invariance factors will be presented. The analysis in the first part of this section will be restricted to the following single, first order $\mathrm{O} \Delta \mathrm{E}$ (see also [1]):

$x_{n+1}=f\left(x_{n}, n ; \varepsilon\right)$,

where $\varepsilon$ is a small parameter. In most applications the function $f$ has the form

$$
\begin{aligned}
f\left(x_{n}, n ; \varepsilon\right)= & f_{0}\left(x_{n}, n\right)+\varepsilon f_{1}\left(x_{n}, n\right) \\
& +\varepsilon^{2} f_{2}\left(x_{n}, n\right)+\cdots .
\end{aligned}
$$

An invariance factor $\mu\left(x_{n}, n ; \varepsilon\right)$ for (7) has to satisfy (5). Now it will also be assumed that $\mu$ can be expanded in a power series in $\varepsilon$, that is,

$$
\begin{aligned}
\mu\left(x_{n}, n ; \varepsilon\right)= & \mu_{0}\left(x_{n}, n\right)+\varepsilon \mu_{1}\left(x_{n}, n\right) \\
& +\varepsilon^{2} \mu_{2}\left(x_{n}, n\right)+\cdots .
\end{aligned}
$$

The expansions (8) and (9) are then substituted into (5), yielding

$$
\begin{aligned}
\left\{\mu_{0}\left(x_{n}, n\right)+\varepsilon \mu_{1}\left(x_{n}, n\right)+\cdots\right\} x_{n} \\
=\left\{\mu_{0}\left(f_{0}\left(x_{n}, n\right)+\varepsilon f_{1}\left(x_{n}, n\right)+\cdots, n+1\right)\right. \\
\quad+\varepsilon \mu_{1}\left(f_{0}\left(x_{n}, n\right)+\varepsilon f_{1}\left(x_{n}, n\right)+\cdots, n+1\right) \\
\quad+\cdots\}\left(f_{0}\left(x_{n}, n\right)+\varepsilon f_{1}\left(x_{n}, n\right)+\cdots\right) .
\end{aligned}
$$

Now it should be observed that (for $i=0,1,2, \ldots$ )

$$
\begin{aligned}
\mu_{i}\left(f_{0}\right. & \left.+\varepsilon f_{1}+\varepsilon^{2} f_{2}+\cdots, n+1\right) \\
= & \mu_{i}\left(f_{0}, n+1\right)+\varepsilon \mu_{i}^{\prime}\left(f_{0}, n+1\right) f_{1} \\
& +\varepsilon^{2}\left[\left(\mu_{i}^{\prime}\left(f_{0}, n+1\right) f_{2}+\frac{1}{2} \mu_{i}^{\prime \prime}\left(f_{0}, n+1\right) f_{1}^{2}\right]\right. \\
& +\varepsilon^{3}(\cdots)+\cdots,
\end{aligned}
$$

where the prime ' denotes differentiation with respect to the first argument of $\mu_{i}$. Then by using (11) and by taking apart in $(10)$ the $O(1)$-terms, the $O(\varepsilon)$-terms, the $O\left(\varepsilon^{2}\right)$-terms, and so on, it follows that the $O(1)$ problem becomes

$\mu_{0}\left(x_{n}, n\right) x_{n}=\mu_{0}\left(f_{0}\left(x_{n}, n\right), n+1\right) f_{0}\left(x_{n}, n\right)$,

that the $O(\varepsilon)$-problem becomes

$$
\begin{aligned}
\mu_{1}\left(x_{n}, n\right) x_{n}= & \mu_{1}\left(f_{0}\left(x_{n}, n\right), n+1\right) f_{0}\left(x_{n}, n\right) \\
& +\mu_{0}\left(f_{0}\left(x_{n}, n\right), n+1\right) f_{1}\left(x_{n}, n\right) \\
& +\mu_{0}^{\prime}\left(f_{0}\left(x_{n}, n\right), n+1\right) \\
& \times f_{1}\left(x_{n}, n\right) f_{0}\left(x_{n}, n\right),
\end{aligned}
$$

and that the $O\left(\varepsilon^{2}\right)$-problem becomes

$$
\begin{aligned}
\mu_{2}\left(x_{n}, n\right) x_{n}= & \mu_{2}\left(f_{0}\left(x_{n}, n\right), n+1\right) f_{0}\left(x_{n}, n\right) \\
& +\mu_{0}\left(f_{0}\left(x_{n}, n\right), n+1\right) f_{2}\left(x_{n}, n\right) \\
& +\mu_{0}^{\prime}\left(f_{0}\left(x_{n}, n\right), n+1\right)\left[f_{1}^{2}\left(x_{n}, n\right)\right. \\
& \left.+f_{2}\left(x_{n}, n\right) f_{0}\left(x_{n}, n\right)\right] \\
& +\frac{1}{2} \mu_{0}^{\prime \prime}\left(f_{0}\left(x_{n}, n\right), n+1\right) f_{1}^{2}\left(x_{n}, n\right) \\
& \times f_{0}\left(x_{n}, n\right)+\mu_{1}\left(f_{0}\left(x_{n}, n\right), n+1\right) \\
& \times f_{1}\left(x_{n}, n\right)+\mu_{1}^{\prime}\left(f_{0}\left(x_{n}, n\right), n+1\right) \\
& \times f_{0}\left(x_{n}, n\right) f_{1}\left(x_{n}, n\right) .
\end{aligned}
$$

For a given function $f\left(x_{n}, n ; \varepsilon\right)$ the $O(1)$-problem, the $O(\varepsilon)$-problem, and so on now have to be solved. For some examples, the reader is referred to [1].

For a system of two first order $\mathrm{O} \Delta \mathrm{Es}$,

$$
\left\{\begin{array}{c}
x_{n+1}=f_{1}\left(x_{n}, y_{n}, n ; \varepsilon\right)=f_{1,0}\left(x_{n}, y_{n}, n\right) \\
\quad+\varepsilon f_{1,1}\left(x_{n}, y_{n}, n\right)+\varepsilon^{2} f_{1,2}\left(x_{n}, y_{n}, n\right)+\cdots, \\
y_{n+1}=f_{2}\left(x_{n}, y_{n}, n ; \varepsilon\right)=f_{2,0}\left(x_{n}, y_{n}, n\right) \\
\quad+\varepsilon f_{2,1}\left(x_{n}, y_{n}, n\right)+\varepsilon^{2} f_{2,2}\left(x_{n}, y_{n}, n\right)+\cdots,
\end{array}\right.
$$

the same procedure can be followed. An invariance vector $\mu\left(x_{n}, y_{n}, n ; \varepsilon\right)$ for (15) has to satisfy (5). Now it will also be assumed that $\mu=\left(\mu_{1}, \mu_{2}\right)^{T}$ can be expanded in a power series in $\bar{\varepsilon}$, that is,

$$
\left\{\begin{array}{l}
\mu_{1}\left(x_{n}, y_{n}, n ; \varepsilon\right)=\mu_{1,0}\left(x_{n}, y_{n}, n\right) \\
\quad+\varepsilon \mu_{1,1}\left(x_{n}, y_{n}, n\right)+\varepsilon^{2} \mu_{1,2}\left(x_{n}, y_{n}, n\right)+\cdots \\
\mu_{2}\left(x_{n}, y_{n}, n ; \varepsilon\right)=\mu_{2,0}\left(x_{n}, y_{n}, n\right) \\
\quad+\varepsilon \mu_{2,1}\left(x_{n}, y_{n}, n\right)+\varepsilon^{2} \mu_{2,2}\left(x_{n}, y_{n}, n\right)+\cdots .
\end{array}\right.
$$

It is obvious from (5) that the functional equation for a system of two first order $\mathrm{O} \Delta$ Es becomes

$$
\begin{aligned}
& \mu_{1}\left(x_{n}, y_{n}, n ; \varepsilon\right) x_{n}+\mu_{2}\left(x_{n}, y_{n}, n ; \varepsilon\right) y_{n} \\
& =\mu_{1}\left(x_{n+1}, y_{n+1}, n+1 ; \varepsilon\right) x_{n+1} \\
& \quad+\mu_{2}\left(x_{n+1}, y_{n+1}, n+1 ; \varepsilon\right) y_{n+1} .
\end{aligned}
$$

After substitution of (15) and of (16) into (17) we will have

$$
\begin{aligned}
\mu_{i, j}( & f_{1,0}+\varepsilon f_{1,1}+\varepsilon^{2} f_{1,2}+\cdots, f_{2,0}+\varepsilon f_{2,1} \\
& \left.+\varepsilon^{2} f_{2,2}+\cdots, n+1\right) \\
= & \mu_{i, j}\left(f_{1,0}, f_{2,0}, n+1\right) \\
& +\varepsilon\left[D_{1}\left(\mu_{i, j}\right)\left(f_{1,0}, f_{2,0}, n+1\right) f_{1,1}\right.
\end{aligned}
$$




$$
\begin{aligned}
& \left.+D_{2}\left(\mu_{i, j}\right)\left(f_{1,0}, f_{2,0}, n+1\right) f_{2,1}\right] \\
& +\varepsilon^{2}\left[D_{2}\left(\mu_{i, j}\right)\left(f_{1,0}, f_{2,0}, n+1\right) f_{2,2}\right. \\
& +D_{1}\left(\mu_{i, j}\right)\left(f_{1,0}, f_{2,0}, n+1\right) f_{1,2} \\
& +D_{1,2}\left(\mu_{i, j}\right)\left(f_{1,0}, f_{2,0}, n+1\right) f_{1,1} f_{2,1} \\
& +\frac{1}{2} D_{1,1}\left(\mu_{i, j}\right)\left(f_{1,0}, f_{2,0}, n+1\right) f_{1,1}^{2}
\end{aligned}
$$

$$
\left.+\frac{1}{2} D_{2,2}\left(\mu_{i, j}\right)\left(f_{1,0}, f_{2,0}, n+1\right) f_{2,1}^{2}\right]+\cdots,
$$

where the subscripts 1 and 2 of $D$ are differentiation with respect to the first and second arguments of $\mu_{i, j}$, respectively, for $i=1,2$ and $j=0,1,2, \ldots$; Then it follows that the $O(1)$-problem becomes

$$
\begin{aligned}
\mu_{1,0}\left(x_{n}, y_{n}, n\right) x_{n}+\mu_{2,0}\left(x_{n}, y_{n}, n\right) y_{n}= & \mu_{1,0}\left(f_{1,0}\left(x_{n}, y_{n}, n\right), f_{2,0}\left(x_{n}, y_{n}, n\right), n+1\right) f_{1,0}\left(x_{n}, y_{n}, n\right) \\
& +\mu_{2,0}\left(f_{1,0}\left(x_{n}, y_{n}, n\right), f_{2,0}\left(x_{n}, y_{n}, n\right), n+1\right) f_{2,0}\left(x_{n}, y_{n}, n\right),
\end{aligned}
$$

and the $O(\varepsilon)$-problem becomes

$$
\begin{aligned}
\mu_{1,1}( & \left.x_{n}, y_{n}, n\right) x_{n}+\mu_{2,1}\left(x_{n}, y_{n}, n\right) y_{n} \\
= & f_{1,1}\left(x_{n}, y_{n}, n\right) \mu_{1,0}\left(f_{1,0}\left(x_{n}, y_{n}, n\right), f_{2,0}\left(x_{n}, y_{n}, n\right), n+1\right) \\
& +f_{1,0}\left(x_{n}, y_{n}, n\right) f_{1,1}\left(x_{n}, y_{n}, n\right) D_{1}\left(\mu_{1,0}\right)\left(f_{1,0}\left(x_{n}, y_{n}, n\right), f_{2,0}\left(x_{n}, y_{n}, n\right), n+1\right) \\
& +f_{1,0}\left(x_{n}, y_{n}, n\right) f_{2,1}\left(x_{n}, y_{n}, n\right) D_{2}\left(\mu_{1,0}\right)\left(f_{1,0}\left(x_{n}, y_{n}, n\right), f_{2,0}\left(x_{n}, y_{n}, n\right), n+1\right) \\
& +f_{1,0}\left(x_{n}, y_{n}, n\right) \mu_{1,1}\left(f_{1,0}\left(x_{n}, y_{n}, n\right), f_{2,0}\left(x_{n}, y_{n}, n\right), n+1\right) \\
& +f_{2,1}\left(x_{n}, y_{n}, n\right) \mu_{2,0}\left(f_{1,0}\left(x_{n}, y_{n}, n\right), f_{2,0}\left(x_{n}, y_{n}, n\right), n+1\right) \\
& +f_{1,1}\left(x_{n}, y_{n}, n\right) f_{2,0}\left(x_{n}, y_{n}, n\right) D_{1}\left(\mu_{2,0}\right)\left(f_{1,0}\left(x_{n}, y_{n}, n\right), f_{2,0}\left(x_{n}, y_{n}, n\right), n+1\right) \\
& +f_{2,0}\left(x_{n}, y_{n}, n\right) f_{2,1}\left(x_{n}, y_{n}, n\right) D_{2}\left(\mu_{2,0}\right)\left(f_{1,0}\left(x_{n}, y_{n}, n\right), f_{2,0}\left(x_{n}, y_{n}, n\right), n+1\right) \\
& +f_{2,0}\left(x_{n}, y_{n}, n\right) \mu_{2,1}\left(f_{1,0}\left(x_{n}, y_{n}, n\right), f_{2,0}\left(x_{n}, y_{n}, n\right), n+1\right) .
\end{aligned}
$$

In the next two sections it will be shown how the perturbation method can be applied to a system of two first order $\mathrm{O} \Delta$ Es. In Sect. 4 a system of two linear equations will be considered, and in Sect. 5 a system of two weakly nonlinear $\mathrm{O} \Delta$ Es will be studied.

\section{A system of two linear, first order $\mathrm{O} \Delta \mathrm{Es}$}

To show how the method can be applied in practice, we will consider the following differential equation:

$\ddot{x}+\mu \dot{x}+x=0$,

where $x=x(t)$, and where $\mu$ is a small, positive damping parameter. This leads to the following dif- ference equation when a central difference scheme is used to discretize (21):

$\frac{x_{n+1}-2 x_{n}+x_{n-1}}{h^{2}}+\mu \frac{x_{n+1}-x_{n-1}}{2 h}+x_{n}=0$,

or equivalently,

$x_{n+1}+\left(h^{2}-2\right) x_{n}+x_{n-1}=\frac{\mu h}{2}\left(x_{n-1}-x_{n+1}\right)$.

In fact, (23) can be considered as a central finite difference approximation of (21). In this case, $x_{n}$ is an approximation of $x\left(t_{n}\right)$ at $t_{n}=n h$, where $h$ is the discretization time step. Now let

$\left\{\begin{array}{l}h^{2}-2=-2 \cos \left(\theta_{0}\right), \\ \frac{\mu h}{2}=\varepsilon .\end{array}\right.$ 
Substitution of these new constants $\theta_{0}$ and $\varepsilon$ from (24) into (23) and shifting the index by 1 , yields

$x_{n+2}-2 \cos \left(\theta_{0}\right) x_{n+1}+x_{n}=\varepsilon\left(x_{n}-x_{n+2}\right)$.

Now (25) is transformed into a system of first order difference equations,

$\left\{\begin{array}{l}x_{n+1}=y_{n}, \\ y_{n+1}=2 y_{n} \cos \left(\theta_{0}\right)-x_{n}+\varepsilon\left(x_{n}-y_{n+1}\right),\end{array}\right.$

or equivalently,

$\left\{\begin{array}{l}x_{n+1}=y_{n} \\ y_{n+1}=\frac{1}{1+\varepsilon}\left(2 y_{n} \cos \left(\theta_{0}\right)-x_{n}+\varepsilon x_{n}\right)\end{array}\right.$

and after expanding $(1+\varepsilon)^{-1}$ into $1-\varepsilon+O\left(\varepsilon^{2}\right)$ for $\varepsilon$ small, it follows that (27) becomes

$$
\left\{\begin{array}{l}
x_{n+1}=y_{n}, \\
y_{n+1}=2 y_{n} \cos \left(\theta_{0}\right)-x_{n}+2 \varepsilon\left(x_{n}-y_{n} \cos \left(\theta_{0}\right)\right) \\
\quad+O\left(\varepsilon^{2}\right) .
\end{array}\right.
$$

Then we substitute (28) into (15) and (19). So, the $O$ (1)-problem will be

$$
\begin{aligned}
\mu_{1,0} & \left(x_{n}, y_{n}, n\right) x_{n}+\mu_{2,0}\left(x_{n}, y_{n}, n\right) y_{n} \\
= & \mu_{1,0}\left(y_{n}, 2 y_{n} \cos \left(\theta_{0}\right)-x_{n}, n+1\right) y_{n} \\
& +\mu_{2,0}\left(y_{n}, 2 y_{n} \cos \left(\theta_{0}\right)-x_{n}, n+1\right) \\
& \times\left(2 y_{n} \cos \left(\theta_{0}\right)-x_{n}\right) .
\end{aligned}
$$

Now we define a new function

$$
\begin{aligned}
Z_{0}\left(x_{n}, y_{n}, n\right)= & \mu_{1,0}\left(x_{n}, y_{n}, n\right) x_{n} \\
& +\mu_{2,0}\left(x_{n}, y_{n}, n\right) y_{n},
\end{aligned}
$$

and by using (30), (29) becomes

$Z_{0}\left(x_{n}, y_{n}, n\right)=Z_{0}\left(y_{n}, 2 y_{n} \cos \left(\theta_{0}\right)-x_{n}, n+1\right)$.

According to the results as obtained in Appendix, the general solution of (31) is given by

$$
\begin{aligned}
& Z_{0}\left(x_{n}, y_{n}, n\right) \\
& =A_{0}\left(x_{n}^{2}-2 x_{n} y_{n} \cos \left(\theta_{0}\right)+y_{n}^{2},\right. \\
& \left.\quad n+\frac{1}{\theta_{0}} \arctan \left(\frac{y_{n} \sin \left(\theta_{0}\right)}{x_{n}-y_{n} \cos \left(\theta_{0}\right)}\right)\right),
\end{aligned}
$$

where $A_{0}$ is an arbitrary function. For more information regarding how to solve the functional equation (31), we refer the reader to Appendix and [15].
According to (30) and (32), we now have

$$
\begin{gathered}
\mu_{1,0}\left(x_{n}, y_{n}, n\right) x_{n}+\mu_{2,0}\left(x_{n}, y_{n}, n\right) y_{n} \\
=A_{0}\left(x_{n}^{2}-2 x_{n} y_{n} \cos \left(\theta_{0}\right)+y_{n}^{2},\right. \\
\left.n+\frac{1}{\theta_{0}} \arctan \left(\frac{y_{n} \sin \left(\theta_{0}\right)}{x_{n}-y_{n} \cos \left(\theta_{0}\right)}\right)\right) .
\end{gathered}
$$

Since we are dealing with a second order difference equation (25), we need two functionally independent approximations of the invariants. The function $A_{0}$ is still arbitrary, and will now be chosen to be as simple as possible to obtain relatively simple approximations of the invariants.

\subsection{Case 1}

First, we take

$\left\{\begin{array}{l}A_{0} \equiv x_{n}^{2}-2 x_{n} y_{n} \cos \left(\theta_{0}\right)+y_{n}^{2}, \\ \mu_{1,0}\left(x_{n}, y_{n}, n\right)=0 .\end{array}\right.$

So, from (30) and (33), we can find

$\mu_{2,0}=\frac{1}{y_{n}}\left(x_{n}^{2}-2 x_{n} y_{n} \cos \left(\theta_{0}\right)+y_{n}^{2}\right)$.

By substituting the values of $\mu_{1,0}$ and $\mu_{2,0}$ into (20), the $O(\varepsilon)$-problem then becomes

$$
\begin{aligned}
\mu_{1,1}( & \left.x_{n}, y_{n}, n\right) x_{n}+\mu_{2,1}\left(x_{n}, y_{n}, n\right) y_{n} \\
= & \mu_{1,1}\left(y_{n}, 2 y_{n} \cos \left(\theta_{0}\right)-x_{n}, n+1\right) y_{n} \\
& +\mu_{2,1}\left(y_{n}, 2 y_{n} \cos \left(\theta_{0}\right)-x_{n}, n+1\right) \\
& \quad \times\left(2 y_{n} \cos \left(\theta_{0}\right)-x_{n}\right)-4\left(x_{n}-y_{n} \cos \left(\theta_{0}\right)\right)^{2},
\end{aligned}
$$

or equivalently,

$$
\begin{aligned}
Z_{1}\left(x_{n}, y_{n}, n\right)= & Z_{1}\left(y_{n}, 2 y_{n} \cos \left(\theta_{0}\right)-x_{n}, n+1\right) \\
& -4\left(x_{n}-y_{n} \cos \left(\theta_{0}\right)\right)^{2}
\end{aligned}
$$

where $Z_{1}\left(x_{n}, y_{n}, n\right)=\mu_{1,1}\left(x_{n}, y_{n}, n\right) x_{n}+\mu_{2,1}\left(x_{n}\right.$, $\left.y_{n}, n\right) y_{n}$. When we put $\mu_{2,0}=0$ instead of $\mu_{1,0}=0$, then $\mu_{1,0}=\frac{1}{x_{n}}\left(x_{n}^{2}-2 x_{n} y_{n} \cos \left(\theta_{0}\right)+y_{n}^{2}\right)$. The interesting point is that the $O(\varepsilon)$-problem then also leads to (37). According to (31) and (32), the general solution $Z_{1, h}$ of the equation corresponding to the homogeneous equation (37) is 


$$
\begin{aligned}
& Z_{1, h}\left(x_{n}, y_{n}, n\right) \\
& =A_{1}\left(x_{n}^{2}-2 x_{n} y_{n} \cos \left(\theta_{0}\right)+y_{n}^{2},\right. \\
& \left.\quad n+\frac{1}{\theta_{0}} \arctan \left(\frac{y_{n} \sin \left(\theta_{0}\right)}{x_{n}-y_{n} \cos \left(\theta_{0}\right)}\right)\right) .
\end{aligned}
$$

Now we are going to construct a particular solution of (37). To do this, we look for a particular solution in the form

$$
Z_{1, p}\left(x_{n}, y_{n}, n\right)=a(n) x_{n}^{2}+b(n) x_{n} y_{n}+c(n) y_{n}^{2} \text {. }
$$

By substituting (39) into (37), we obtain

$$
\begin{aligned}
& a(n) x_{n}^{2}+b(n) x_{n} y_{n}+c(n) y_{n}^{2} \\
& =a(n+1) y_{n}^{2}+b(n+1) y_{n}\left(2 y_{n} \cos \left(\theta_{0}\right)-x_{n}\right) \\
& \quad+c(n+1)\left(2 y_{n} \cos \left(\theta_{0}\right)-x_{n}\right)^{2} \\
& \quad-4\left(x_{n}-y_{n} \cos \left(\theta_{0}\right)\right)^{2}
\end{aligned}
$$

or equivalently,

$$
\begin{aligned}
& \left\{a(n+1)-4 \cos ^{2}\left(\theta_{0}\right) a(n)-2 \cos \left(\theta_{0}\right) b(n)-c(n)\right. \\
& \left.\quad-4 \cos ^{2}\left(\theta_{0}\right)\right\} x_{n}^{2}+\left\{b(n+1)+4 \cos \left(\theta_{0}\right) a(n)\right. \\
& \left.\quad+b(n)+8 \cos \left(\theta_{0}\right)\right\} x_{n} y_{n}+\{c(n+1)-a(n) \\
& \quad-4\} y_{n}^{2}=0 .
\end{aligned}
$$

Obviously, the coefficients of $x_{n}^{2}, x_{n} y_{n}$, and $y_{n}^{2}$ have to be zero, yielding

$$
\left\{\begin{array}{l}
a(n+1)=4 \cos ^{2}\left(\theta_{0}\right) a(n)+2 \cos \left(\theta_{0}\right) b(n) \\
\quad+c(n)+4 \cos ^{2}\left(\theta_{0}\right), \\
b(n+1)=-4 \cos \left(\theta_{0}\right) a(n)-b(n)-8 \cos \left(\theta_{0}\right), \\
c(n+1)=a(n)+4 .
\end{array}\right.
$$

The eigenvalues of the homogeneous system related to (42) are 1 , and $\cos \left(2 \theta_{0}\right) \pm i \sin \left(2 \theta_{0}\right)$, and a particular solution of (42) is given by

$$
\left\{\begin{array}{l}
a(n)=S_{1}\left\{(2 n-1) \sin \left(2 \theta_{0}\right)+\sin \left(2 n \theta_{0}\right)\right. \\
\left.\quad+\sin \left(2(n+1) \theta_{0}\right)\right\} \\
b(n)=-4 S_{1} \cos \left(\theta_{0}\right)\left\{n \sin \left(2 \theta_{0}\right)+\sin \left(2 n \theta_{0}\right)\right\} \\
c(n)=S_{1}\left\{(2 n+1) \sin \left(2 \theta_{0}\right)+\sin \left(2 n \theta_{0}\right)\right. \\
\left.\quad+\sin \left(2(n-1) \theta_{0}\right)\right\}
\end{array}\right.
$$

where $S_{1}=\frac{1}{\sin \left(2 \theta_{0}\right)}$. Therefore, according to (39) and (43), a particular solution of (37) is given by

$$
\begin{aligned}
Z_{1, p}( & \left.x_{n}, y_{n}, n\right) \\
= & S_{1}\left\{\left[(2 n-1) \sin \left(2 \theta_{0}\right)+\sin \left(2 n \theta_{0}\right)\right.\right. \\
& \left.+\sin \left(2(n+1) \theta_{0}\right)\right] x_{n}^{2}-4 \cos \left(\theta_{0}\right)\left[n \sin \left(2 \theta_{0}\right)\right. \\
& \left.+\sin \left(2 n \theta_{0}\right)\right] x_{n} y_{n}+\left[(2 n+1) \sin \left(2 \theta_{0}\right)\right. \\
& \left.\left.+\sin \left(2 n \theta_{0}\right)+\sin \left(2(n-1) \theta_{0}\right)\right] y_{n}^{2}\right\}
\end{aligned}
$$

So, from (36)-(38), we have

$$
\begin{aligned}
& \mu_{1,1}\left(x_{n}, y_{n}, n\right) x_{n}+\mu_{2,1}\left(x_{n}, y_{n}, n\right) y_{n} \\
& \quad=Z_{1, h}\left(x_{n}, y_{n}, n\right)+Z_{1, p}\left(x_{n}, y_{n}, n\right),
\end{aligned}
$$

where $Z_{1, h}\left(x_{n}, y_{n}, n\right)$ and $Z_{1, p}\left(x_{n}, y_{n}, n\right)$ are defined in (38) and (44), respectively. We will now choose the invariant as simple as possible, that is, $A_{1} \equiv 0$ in (45), and so for this case we have

$$
\begin{aligned}
\mu_{1,1}( & \left.x_{n}, y_{n}, n\right) x_{n}+\mu_{2,1}\left(x_{n}, y_{n}, n\right) y_{n} \\
= & S_{1}\left\{\left[(2 n-1) \sin \left(2 \theta_{0}\right)+\sin \left(2 n \theta_{0}\right)\right.\right. \\
& \left.+\sin \left(2(n+1) \theta_{0}\right)\right] x_{n}^{2}-4 \cos \left(\theta_{0}\right)\left[n \sin \left(2 \theta_{0}\right)\right. \\
& \left.+\sin \left(2 n \theta_{0}\right)\right] x_{n} y_{n}+\left[(2 n+1) \sin \left(2 \theta_{0}\right)\right. \\
& \left.\left.+\sin \left(2 n \theta_{0}\right)+\sin \left(2(n-1) \theta_{0}\right)\right] y_{n}^{2}\right\}
\end{aligned}
$$

An approximation $I_{A}\left(x_{n}, y_{n}, n\right)$ of an invariant $I\left(x_{n}\right.$, $\left.y_{n}, n\right)=$ constant for (28) is now given by (see also (6)):

$$
\begin{aligned}
I_{A}\left(x_{n}, y_{n}, n\right)= & \mu_{1,0}\left(x_{n}, y_{n}, n\right) x_{n}+\mu_{2,0}\left(x_{n}, y_{n}, n\right) y_{n} \\
& +\varepsilon\left\{\mu_{1,1}\left(x_{n}, y_{n}, n\right) x_{n}\right. \\
& \left.+\mu_{2,1}\left(x_{n}, y_{n}, n\right) y_{n}\right\} .
\end{aligned}
$$

Then by using (33), (34), and (46), we conclude that

$$
\begin{aligned}
I_{A}\left(x_{n}, y_{n}, n\right) \\
=x_{n}^{2}-2 x_{n} y_{n} \cos \left(\theta_{0}\right)+y_{n}^{2} \\
\quad+\varepsilon S_{1}\left\{\left[(2 n-1) \sin \left(2 \theta_{0}\right)+\sin \left(2 n \theta_{0}\right)\right.\right. \\
\left.\quad+\sin \left(2(n+1) \theta_{0}\right)\right] x_{n}^{2}-4 \cos \left(\theta_{0}\right)\left[n \sin \left(2 \theta_{0}\right)\right. \\
\left.\quad+\sin \left(2 n \theta_{0}\right)\right] x_{n} y_{n}+\left[(2 n+1) \sin \left(2 \theta_{0}\right)\right. \\
\left.\left.\quad+\sin \left(2 n \theta_{0}\right)+\sin \left(2(n-1) \theta_{0}\right)\right] y_{n}^{2}\right\},
\end{aligned}
$$

and

$$
\begin{aligned}
I_{A}\left(x_{n+1}, y_{n+1}, n+1\right)= & I_{A}\left(x_{n}, y_{n}, n\right) \\
& +\varepsilon^{2} R\left(x_{n}, y_{n}, n ; \varepsilon\right),
\end{aligned}
$$


where

$$
\begin{aligned}
\varepsilon^{2} R & \left(x_{n}, y_{n}, n ; \varepsilon\right) \\
= & -2 f_{1}\left(f_{2}-f_{2,0}-\varepsilon f_{2,1}\right) \cos \left(\theta_{0}\right) \\
& +\left(f_{2}^{2}-f_{2,0}^{2}-2 \varepsilon f_{2,0} f_{2,1}\right)+\varepsilon\{b(n+1) \\
& \left.\times f_{1}\left(f_{2}-f_{2,0}\right)+c(n+1)\left(f_{2}^{2}-f_{2,0}^{2}\right)\right\},
\end{aligned}
$$

where $f_{1}, f_{2}, f_{2,0}$ and $f_{2,1}$ are given by (15) and (28). Since the system (28) is $n$-independent, that is, $f_{1}$ and $f_{2}$ are $n$-independent, and $b(n)$ and $c(n)$ are of $O(n)$, then $R$ is unbounded in $n$ and of $O(n)$. From (49), it follows that

$$
\begin{aligned}
I_{A}\left(x_{n}, y_{n}, n\right)= & I_{A}\left(x_{0}, y_{0}, 0\right) \\
& +\varepsilon^{2} \sum_{i=0}^{n-1} R\left(x_{i}, y_{i}, i ; \varepsilon\right) .
\end{aligned}
$$

From (48)-(51), it can be shown that

$$
\begin{aligned}
I_{A}\left(x_{n}, y_{n}, n\right)= & x_{0}^{2}+y_{0}^{2}-2 x_{0} y_{0} \cos \left(\theta_{0}\right) \\
& +O\left(\varepsilon^{2} n^{2}\right),
\end{aligned}
$$

it then follows that

$$
\left\{\begin{array}{l}
I_{A}\left(x_{n}, y_{n}, n\right)=\text { constant }+O\left(\varepsilon^{2}\right) \\
\quad \text { for } n=O(1), \\
I_{A}\left(x_{n}, y_{n}, n\right)=\text { constant }+O(\varepsilon) \\
\quad \text { for } n=O\left(\frac{1}{\sqrt{\varepsilon}}\right) .
\end{array}\right.
$$

So far, only one approximation of a first integral has been determined. Another (functionally independent) approximation of a first integral can also be obtained in a similar and straightforward way as follows from the next subsection.

\subsection{Case 2}

Now we take

$$
\left\{\begin{array}{l}
A_{0} \equiv n+\frac{1}{\theta_{0}} \arctan \left(\frac{y_{n} \sin \left(\theta_{0}\right)}{x_{n}-y_{n} \cos \left(\theta_{0}\right)}\right), \\
\mu_{1,0}\left(x_{n}, y_{n}, n\right)=0 .
\end{array}\right.
$$

So, from (30) and (33), we can find

$$
\mu_{2,0}=\frac{1}{y_{n}}\left(n+\frac{1}{\theta_{0}} \arctan \left(\frac{y_{n} \sin \left(\theta_{0}\right)}{x_{n}-y_{n} \cos \left(\theta_{0}\right)}\right)\right) .
$$

By substituting the values of $\mu_{1,0}$ and $\mu_{2,0}$ into (20), the $O(\varepsilon)$-problem then becomes

$$
\begin{aligned}
\mu_{1,1} & \left(x_{n}, y_{n}, n\right) x_{n}+\mu_{2,1}\left(x_{n}, y_{n}, n\right) y_{n} \\
= & \mu_{1,1}\left(y_{n}, 2 y_{n} \cos \left(\theta_{0}\right)-x_{n}, n+1\right) y_{n} \\
& +\mu_{2,1}\left(y_{n}, 2 y_{n} \cos \left(\theta_{0}\right)-x_{n}, n+1\right) \\
& \times\left(2 y_{n} \cos \left(\theta_{0}\right)-x_{n}\right) \\
& +\frac{2 y_{n}\left(x_{n}-y_{n} \cos \left(\theta_{0}\right)\right) \sin \left(\theta_{0}\right)}{\theta_{0}\left(x_{n}^{2}-2 x_{n} y_{n} \cos \left(\theta_{0}\right)+y_{n}^{2}\right)},
\end{aligned}
$$

or equivalently,

$$
\begin{aligned}
& Z_{1}\left(x_{n}, y_{n}, n\right) \\
& =Z_{1}\left(y_{n}, 2 y_{n} \cos \left(\theta_{0}\right)-x_{n}, n+1\right) \\
& \quad+\frac{2 y_{n}\left(x_{n}-y_{n} \cos \left(\theta_{0}\right)\right) \sin \left(\theta_{0}\right)}{\theta_{0}\left(x_{n}^{2}-2 x_{n} y_{n} \cos \left(\theta_{0}\right)+y_{n}^{2}\right)},
\end{aligned}
$$

where $Z_{1}\left(x_{n}, y_{n}, n\right)=\mu_{1,1}\left(x_{n}, y_{n}, n\right) x_{n}+\mu_{2,1}\left(x_{n}\right.$, $\left.y_{n}, n\right) y_{n}$. When we put $\mu_{2,0}=0$ instead of $\mu_{1,0}=0$, then $\mu_{1,0}=\frac{1}{x_{n}}\left(n+\frac{1}{\theta_{0}} \arctan \left(\frac{y_{n} \sin \left(\theta_{0}\right)}{x_{n}-y_{n} \cos \left(\theta_{0}\right)}\right)\right)$. The interesting point is that again the $O(\varepsilon)$-problem leads to (57). According to (31) and (32), the general solution $Z_{1, h}$ of the equation corresponding to the homogeneous equation (57) is

$$
\begin{aligned}
& Z_{1, h}\left(x_{n}, y_{n}, n\right) \\
& =A_{1}\left(x_{n}^{2}-2 x_{n} y_{n} \cos \left(\theta_{0}\right)+y_{n}^{2},\right. \\
& \left.\quad n+\frac{1}{\theta_{0}} \arctan \left(\frac{y_{n} \sin \left(\theta_{0}\right)}{x_{n}-y_{n} \cos \left(\theta_{0}\right)}\right)\right) .
\end{aligned}
$$

Now we are going to construct a particular solution of (57). Since

$$
\frac{2 \sin \left(\theta_{0}\right)}{\theta_{0}\left(x_{n}^{2}-2 x_{n} y_{n} \cos \left(\theta_{0}\right)+y_{n}^{2}\right)}
$$

satisfies the homogeneous equation related to (57), we consider a particular solution of (57) in the form

$$
\begin{aligned}
& Z_{1, p}\left(x_{n}, y_{n}, n\right) \\
& =\frac{2 \sin \left(\theta_{0}\right)}{\theta_{0}\left(x_{n}^{2}-2 x_{n} y_{n} \cos \left(\theta_{0}\right)+y_{n}^{2}\right)} \\
& \quad \times\left\{a(n) x_{n}^{2}+b(n) x_{n} y_{n}+c(n) y_{n}^{2}\right\} .
\end{aligned}
$$

By substituting (59) into (57), we obtain 


$$
\begin{aligned}
& a(n) x_{n}^{2}+b(n) x_{n} y_{n}+c(n) y_{n}^{2} \\
& =a(n+1) y_{n}^{2}+b(n+1) y_{n}\left(2 y_{n} \cos \left(\theta_{0}\right)-x_{n}\right) \\
& \quad+c(n+1)\left(2 y_{n} \cos \left(\theta_{0}\right)-x_{n}\right)^{2} \\
& \quad+y_{n}\left(x_{n}-y_{n} \cos \left(\theta_{0}\right)\right),
\end{aligned}
$$

or equivalently,

$$
\begin{aligned}
& \left\{a(n+1)-4 \cos ^{2}\left(\theta_{0}\right) a(n)-2 \cos \left(\theta_{0}\right) b(n)-c(n)\right. \\
& \left.\quad+\cos \left(\theta_{0}\right)\right\} x_{n}^{2}+\left\{b(n+1)+4 \cos \left(\theta_{0}\right) a(n)\right. \\
& \quad+b(n)-1\} x_{n} y_{n}+\{c(n+1)-a(n)\} y_{n}^{2}=0 .
\end{aligned}
$$

Obviously, the coefficients of $x_{n}^{2}, x_{n} y_{n}$, and $y_{n}^{2}$ have to be zero, yielding

$$
\left\{\begin{array}{l}
a(n+1)=4 \cos ^{2}\left(\theta_{0}\right) a(n)+2 \cos \left(\theta_{0}\right) b(n) \\
\quad+c(n)-\cos \left(\theta_{0}\right), \\
b(n+1)=-4 \cos \left(\theta_{0}\right) a(n)-b(n)+1, \\
c(n+1)=a(n) .
\end{array}\right.
$$

The eigenvalues of the homogeneous system related to (62) are 1 , and $\cos \left(2 \theta_{0}\right) \pm i \sin \left(2 \theta_{0}\right)$, and a particular solution of (62) is given by

$$
\left\{\begin{array}{l}
a(n)=S_{2}\left\{\cos \left((2 n+1) \theta_{0}\right)-\cos \left(\theta_{0}\right)\right\}, \\
b(n)=4 S_{2} \sin ^{2}\left(n \theta_{0}\right), \\
c(n)=S_{2}\left\{\cos \left((2 n-1) \theta_{0}\right)-\cos \left(\theta_{0}\right)\right\},
\end{array}\right.
$$

where $S_{2}=\frac{1}{4 \sin ^{2}\left(\theta_{0}\right)}$. Therefore, according to (59) and (63), a particular solution of (57) is given by

$$
\begin{aligned}
& Z_{1, p}\left(x_{n}, y_{n}, n\right) \\
&= \frac{2 S_{2} \sin \left(\theta_{0}\right)}{\theta_{0}\left(x_{n}^{2}-2 x_{n} y_{n} \cos \left(\theta_{0}\right)+y_{n}^{2}\right)} \\
& \quad \times\left\{x_{n}^{2}\left[\cos \left((2 n+1) \theta_{0}\right)-\cos \left(\theta_{0}\right)\right]+4 x_{n} y_{n}\right. \\
&\left.\quad \times \sin ^{2}\left(n \theta_{0}\right)+y_{n}^{2}\left[\cos \left((2 n-1) \theta_{0}\right)-\cos \left(\theta_{0}\right)\right]\right\} .
\end{aligned}
$$

So, from (56)-(58), we have

$$
\begin{gathered}
\mu_{1,1}\left(x_{n}, y_{n}, n\right) x_{n}+\mu_{2,1}\left(x_{n}, y_{n}, n\right) y_{n} \\
=A_{1}\left(x_{n}^{2}-2 x_{n} y_{n} \cos \left(\theta_{0}\right)+y_{n}^{2},\right. \\
\left.n+\frac{1}{\theta_{0}} \arctan \left(\frac{y_{n} \sin \left(\theta_{0}\right)}{x_{n}-y_{n} \cos \left(\theta_{0}\right)}\right)\right) \\
+\frac{2 S_{2} \sin \left(\theta_{0}\right)}{\theta_{0}\left(x_{n}^{2}-2 x_{n} y_{n} \cos \left(\theta_{0}\right)+y_{n}^{2}\right)}
\end{gathered}
$$

$$
\begin{aligned}
& \times\left\{x_{n}^{2}\left[\cos \left((2 n+1) \theta_{0}\right)-\cos \left(\theta_{0}\right)\right]\right. \\
& +4 x_{n} y_{n} \sin ^{2}\left(n \theta_{0}\right)+y_{n}^{2}\left[\cos \left((2 n-1) \theta_{0}\right)\right. \\
& \left.\left.-\cos \left(\theta_{0}\right)\right]\right\} .
\end{aligned}
$$

The function $A_{1}$ is still arbitrary but will be chosen to be as simple as possible: $A_{1} \equiv 0$, yielding

$$
\begin{aligned}
\mu_{1,1} & \left(x_{n}, y_{n}, n\right) x_{n}+\mu_{2,1}\left(x_{n}, y_{n}, n\right) y_{n} \\
= & \frac{2 S_{2} \sin \left(\theta_{0}\right)}{\theta_{0}\left(x_{n}^{2}-2 x_{n} y_{n} \cos \left(\theta_{0}\right)+y_{n}^{2}\right)} \\
& \times\left\{x_{n}^{2}\left[\cos \left((2 n+1) \theta_{0}\right)-\cos \left(\theta_{0}\right)\right]\right. \\
& +4 x_{n} y_{n} \sin ^{2}\left(n \theta_{0}\right)+y_{n}^{2}\left[\cos \left((2 n-1) \theta_{0}\right)\right. \\
& \left.\left.-\cos \left(\theta_{0}\right)\right]\right\} .
\end{aligned}
$$

As for the case 1, we can construct an exact difference equation of (28) up to $O\left(\varepsilon^{2}\right)$, in an almost similar way, yielding

$$
\begin{aligned}
& I_{A}\left(x_{n+1}, y_{n+1}, n+1\right) \\
& \quad=I_{A}\left(x_{n}, y_{n}, n\right)+\varepsilon^{2} R\left(x_{n}, y_{n}, n ; \varepsilon\right),
\end{aligned}
$$

where

$$
\begin{aligned}
& I_{A}\left(x_{n}, y_{n}, n ; \varepsilon\right) \\
& =n+\frac{1}{\theta_{0}} \arctan \left(\frac{y_{n} \sin \left(\theta_{0}\right)}{x_{n}-y_{n} \cos \left(\theta_{0}\right)}\right) \\
& \quad+\frac{2 \varepsilon S_{2} \sin \left(\theta_{0}\right)}{\theta_{0}\left(x_{n}^{2}-2 x_{n} y_{n} \cos \left(\theta_{0}\right)+y_{n}^{2}\right)} \\
& \quad \times\left\{x_{n}^{2}\left[\cos \left((2 n+1) \theta_{0}\right)-\cos \left(\theta_{0}\right)\right]\right. \\
& \quad+4 x_{n} y_{n} \sin ^{2}\left(n \theta_{0}\right)+y_{n}^{2}\left[\cos \left((2 n-1) \theta_{0}\right)\right. \\
& \left.\left.\quad-\cos \left(\theta_{0}\right)\right]\right\},
\end{aligned}
$$

and

$$
\begin{aligned}
\varepsilon^{2} R & \left(x_{n}, y_{n}, n ; \varepsilon\right) \\
= & \frac{1}{\theta_{0}}\left\{\arctan \left(\frac{f_{2} \sin \left(\theta_{0}\right)}{f_{1}-f_{2} \cos \left(\theta_{0}\right)}\right)\right. \\
& -\arctan \left(\frac{f_{2,0} \sin \left(\theta_{0}\right)}{f_{1}-f_{2,0} \cos \left(\theta_{0}\right)}\right) \\
& \left.-\frac{f_{1} f_{2,1} \sin \left(\theta_{0}\right)}{f_{1}^{2}-2 f_{1} f_{2,0} \cos \left(\theta_{0}\right)+f_{2,0}^{2}} \varepsilon\right\}+\frac{2 \varepsilon \sin \left(\theta_{0}\right)}{\theta_{0}} \\
& \times\left\{\frac{a(n+1) f_{1}^{2}+b(n+1) f_{1} f_{2}+c(n+1) f_{2}^{2}}{f_{1}^{2}-2 f_{1} f_{2} \cos \left(\theta_{0}\right)+f_{2}^{2}}\right.
\end{aligned}
$$




$$
\left.-\frac{a(n+1) f_{1}^{2}+b(n+1) f_{1} f_{2,0}+c(n+1) f_{2,0}^{2}}{f_{1}^{2}-2 f_{1} f_{2,0} \cos \left(\theta_{0}\right)+f_{2,0}^{2}}\right\},
$$

where $f_{1}, f_{2}, f_{2,0}$ and $f_{2,1}$ are given by (15) and (28). Since the system (28) is $n$-independent, and $a(n), b(n)$ and $c(n)$ are bounded, then $R$ is bounded. From (67), it follows that

$$
\begin{aligned}
I_{A}\left(x_{n}, y_{n}, n\right)= & I_{A}\left(x_{0}, y_{0}, 0\right) \\
& +\varepsilon^{2} \sum_{i=0}^{n-1} R\left(x_{i}, y_{i}, i ; \varepsilon\right),
\end{aligned}
$$

where $I_{A}\left(x_{0}, y_{0}, 0\right)=\frac{1}{\theta_{0}} \arctan \left(\frac{y_{0} \sin \left(\theta_{0}\right)}{x_{0}-y_{0} \cos \left(\theta_{0}\right)}\right)=$ constant. Finally, it follows from (67)-(70) that

$I_{A}\left(x_{n}, y_{n}, n\right)=$ constant $+O\left(\varepsilon^{2} n\right)$,

it then follows that

$$
\left\{\begin{array}{l}
I_{A}\left(x_{n}, y_{n}, n\right)=\text { constant }+O\left(\varepsilon^{2}\right) \\
\quad \text { for } n=O(1), \\
I_{A}\left(x_{n}, y_{n}, n\right)=\text { constant }+O(\varepsilon) \\
\quad \text { for } n=O\left(\frac{1}{\varepsilon}\right) .
\end{array}\right.
$$

The exact solution of the system of difference equations (27) is

$$
\left\{\begin{aligned}
x_{n} & =c_{1} \cos \left(n \theta_{0}\right)+c_{2} \sin \left(n \theta_{0}\right)-n \varepsilon\left(c_{1} \cos \left(n \theta_{0}\right)\right. \\
& \left.+c_{2} \sin \left(n \theta_{0}\right)\right)+O\left(\varepsilon^{2} n^{2}\right) \\
y_{n} & =x_{n+1}
\end{aligned}\right.
$$

and, therefore, it can readily be verified that $I_{A}\left(x_{n}\right.$, $\left.y_{n}, n\right)$ as given by (48) (or by (68)) satisfies $I_{A}\left(x_{n}\right.$, $\left.y_{n}, n\right)=$ constant $+O\left(\varepsilon^{2} n^{2}\right) \quad\left(\right.$ or $I_{A}\left(x_{n}, y_{n}, n\right)=$ constant $\left.+O\left(\varepsilon^{2} n\right)\right)$.

\section{A weakly nonlinear, regularly perturbed system of two $\mathrm{O \Delta Es}$}

In this section, approximations of first integrals for a second order, weakly nonlinear, regularly perturbed $\mathrm{O} \Delta \mathrm{E}$ with a Van der Pol type of nonlinearity will be considered. The Van der Pol equation $[16,17]$ corresponds to a nonlinear oscillatory system that has both input and output sources of energy. This equation is given by

$\ddot{x}+\omega_{0}^{2} x=\mu\left(1-x^{2}\right) \dot{x}$, where $x=x(t), \mu$ is a nonnegative small parameter, and where $\omega_{0}$ is a bounded constant. This leads to the following difference equation, when a central difference scheme [18] is used to discretize (74):

$$
\begin{gathered}
\frac{x_{n+1}-2 x_{n}+x_{n-1}}{h^{2}}+\omega_{0}^{2} x_{n} \\
=\mu\left(1-x_{n}^{2}\right) \frac{x_{n+1}-x_{n-1}}{2 h},
\end{gathered}
$$

or equivalently,

$$
\begin{aligned}
& x_{n+1}+\left(\omega_{0}^{2} h^{2}-2\right) x_{n}+x_{n-1} \\
& =\frac{\mu h}{2}\left(1-x_{n}^{2}\right)\left(x_{n+1}-x_{n-1}\right) .
\end{aligned}
$$

In fact, (76) can be considered as a central finite difference approximation of (74). In this case, $x_{n}$ is an approximation of $x\left(t_{n}\right)$ at $t_{n}=n h$, where $h$ is the discretization time step. Now let

$\left\{\begin{array}{l}h^{2} \omega_{0}^{2}-2=-2 \cos \left(\theta_{0}\right) \\ \frac{\mu h}{2}=\varepsilon\end{array}\right.$

Substitution of these new constants $\theta_{0}$ and $\varepsilon$ from (77) into (76) and shifting the index by 1 , yields

$$
\begin{aligned}
& x_{n+2}-2 \cos \left(\theta_{0}\right) x_{n+1}+x_{n} \\
& \quad=\varepsilon\left(1-x_{n+1}^{2}\right)\left(x_{n+2}-x_{n}\right),
\end{aligned}
$$

where $\varepsilon$ is a small parameter, that is, $0<\varepsilon \ll 1$, and where $\theta_{0}$ is constant (which is related to the stepsize in making the continuous Van der Pol equation discrete). Now (78) is transformed into a system of first order difference equations,

$$
\left\{\begin{array}{l}
x_{n+1}=y_{n}, \\
y_{n+1}=2 y_{n} \cos \left(\theta_{0}\right)-x_{n} \\
\quad+\varepsilon\left(y_{n+1}-x_{n}\right)\left(1-y_{n}^{2}\right),
\end{array}\right.
$$

or equivalently,

$$
\left\{\begin{array}{l}
x_{n+1}=y_{n} \\
y_{n+1}=\frac{1}{1-\varepsilon\left(1-y_{n}^{2}\right)}\left(2 y_{n} \cos \left(\theta_{0}\right)\right. \\
\left.\quad-x_{n}-\varepsilon x_{n}\left(1-y_{n}^{2}\right)\right)
\end{array}\right.
$$

and after expanding $\left(1-\varepsilon\left(1-y_{n}^{2}\right)\right)^{-1}$ into $1+\varepsilon(1-$ $\left.y_{n}^{2}\right)+O\left(\varepsilon^{2}\right)$, it follows that (80) becomes

$$
\left\{\begin{array}{l}
x_{n+1}=y_{n} \\
y_{n+1}=2 y_{n} \cos \left(\theta_{0}\right)-x_{n}+2 \varepsilon\left(x_{n}-y_{n} \cos \left(\theta_{0}\right)\right) \\
\quad \times\left(y_{n}^{2}-1\right)+O\left(\varepsilon^{2}\right)
\end{array}\right.
$$


Then we substitute (81) into (15) and (19). So, the $O$ (1)-problem will be

$$
\begin{aligned}
\mu_{1,0} & \left(x_{n}, y_{n}, n\right) x_{n}+\mu_{2,0}\left(x_{n}, y_{n}, n\right) y_{n} \\
= & \mu_{1,0}\left(y_{n}, 2 y_{n} \cos \left(\theta_{0}\right)-x_{n}, n+1\right) y_{n} \\
& +\mu_{2,0}\left(y_{n}, 2 y_{n} \cos \left(\theta_{0}\right)-x_{n}, n+1\right) \\
& \quad \times\left(2 y_{n} \cos \left(\theta_{0}\right)-x_{n}\right) .
\end{aligned}
$$

Now we define a new function

$$
\begin{aligned}
Z_{0}\left(x_{n}, y_{n}, n\right)= & \mu_{1,0}\left(x_{n}, y_{n}, n\right) x_{n} \\
& +\mu_{2,0}\left(x_{n}, y_{n}, n\right) y_{n},
\end{aligned}
$$

and by using (83), (82) becomes

$$
Z_{0}\left(x_{n}, y_{n}, n\right)=Z_{0}\left(y_{n}, 2 y_{n} \cos \left(\theta_{0}\right)-x_{n}, n+1\right) .
$$

The obtained functional equation is the same as the functional equation (31). Therefore, the general solution of (84) is given by

$$
\begin{aligned}
& Z_{0}\left(x_{n}, y_{n}, n\right) \\
& =A_{0}\left(x_{n}^{2}-2 x_{n} y_{n} \cos \left(\theta_{0}\right)+y_{n}^{2},\right. \\
& \left.\quad n+\frac{1}{\theta_{0}} \arctan \left(\frac{y_{n} \sin \left(\theta_{0}\right)}{x_{n}-y_{n} \cos \left(\theta_{0}\right)}\right)\right),
\end{aligned}
$$

where $A_{0}$ is an arbitrary function. So, according to (83) and (85), we now have

$$
\begin{gathered}
\mu_{1,0}\left(x_{n}, y_{n}, n\right) x_{n}+\mu_{2,0}\left(x_{n}, y_{n}, n\right) y_{n} \\
=A_{0}\left(x_{n}^{2}-2 x_{n} y_{n} \cos \left(\theta_{0}\right)+y_{n}^{2},\right. \\
\left.n+\frac{1}{\theta_{0}} \arctan \left(\frac{y_{n} \sin \left(\theta_{0}\right)}{x_{n}-y_{n} \cos \left(\theta_{0}\right)}\right)\right) .
\end{gathered}
$$

Since we are dealing with a second order difference equation (78), we need two functionally independent approximations of the invariants. The function $A_{0}$ is still arbitrary, and will now be chosen to be as simple as possible to obtain relatively simple approximations of the invariants.

\subsection{Case 1}

First, we take

$$
\left\{\begin{array}{l}
A_{0} \equiv x_{n}^{2}-2 x_{n} y_{n} \cos \left(\theta_{0}\right)+y_{n}^{2}, \\
\mu_{1,0}\left(x_{n}, y_{n}, n\right)=0 .
\end{array}\right.
$$

So, from (83) and (86), we obtain

$$
\mu_{2,0}=\frac{1}{y_{n}}\left(x_{n}^{2}-2 x_{n} y_{n} \cos \left(\theta_{0}\right)+y_{n}^{2}\right) .
$$

By substituting $\mu_{1,0}$ and $\mu_{2,0}$ into (20), the $O(\varepsilon)$ problem then becomes

$$
\begin{aligned}
Z_{1}\left(x_{n}, y_{n}, n\right)= & Z_{1}\left(y_{n}, 2 y_{n} \cos \left(\theta_{0}\right)-x_{n}, n+1\right) \\
& -4\left(x_{n}-y_{n} \cos \left(\theta_{0}\right)\right)^{2}\left(y_{n}^{2}-1\right),
\end{aligned}
$$

where $Z_{1}\left(x_{n}, y_{n}, n\right)=\mu_{1,1}\left(x_{n}, y_{n}, n\right) x_{n}+\mu_{2,1}\left(x_{n}\right.$, $\left.y_{n}, n\right) y_{n}$. When we put $\mu_{2,0}=0$ instead of $\mu_{1,0}=0$, then $\mu_{1,0}=\frac{1}{x_{n}}\left(x_{n}^{2}-2 x_{n} y_{n} \cos \left(\theta_{0}\right)+y_{n}^{2}\right)$. The interesting point is that the $O(\varepsilon)$-problem leads to (89). According to (84) and (85), the general solution $Z_{1, h}$ of the equation corresponding to the homogeneous equation (89) is

$$
\begin{aligned}
& Z_{1, h}\left(x_{n}, y_{n}, n\right) \\
& =A_{1}\left(x_{n}^{2}-2 x_{n} y_{n} \cos \left(\theta_{0}\right)+y_{n}^{2},\right. \\
& \left.\quad n+\frac{1}{\theta_{0}} \arctan \left(\frac{y_{n} \sin \left(\theta_{0}\right)}{x_{n}-y_{n} \cos \left(\theta_{0}\right)}\right)\right) .
\end{aligned}
$$

Now we are going to construct a particular solution of (89). To do this, we look for a particular solution in the form

$$
\begin{aligned}
& Z_{1, p}\left(x_{n}, y_{n}, n\right) \\
& =a(n) x_{n}^{2}+b(n) x_{n} y_{n}+c(n) y_{n}^{2} \\
& \quad+d(n) x_{n}^{4}+e(n) x_{n}^{3} y_{n}+f(n) x_{n}^{2} y_{n}^{2} \\
& \quad+g(n) x_{n} y_{n}^{3}+h(n) y_{n}^{4} .
\end{aligned}
$$

By substituting (91) into (89) and by taking apart in the resulting equation the coefficients of $x_{n}^{2}, x_{n} y_{n}, y_{n}^{2}$, $x_{n}^{4}, x_{n}^{3} y_{n}, x_{n}^{2} y_{n}^{2}, x_{n} y_{n}^{3}$, and $y_{n}^{4}$, and setting these coefficients equal to zero, we will have the following system: 


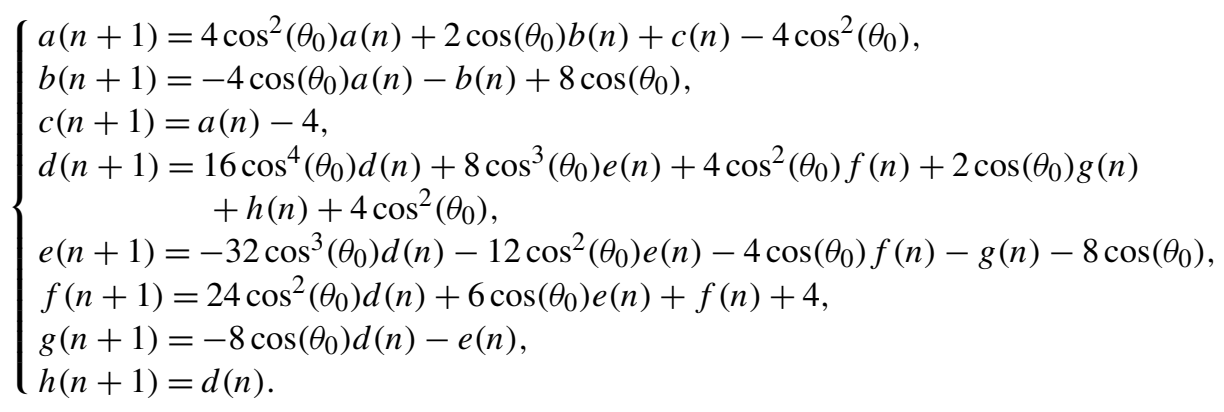

The eigenvalues of the homogeneous system related to (92) are $1,1, \cos \left(2 \theta_{0}\right) \pm i \sin \left(2 \theta_{0}\right), \cos \left(2 \theta_{0}\right) \pm i \sin \left(2 \theta_{0}\right)$, and $\cos \left(4 \theta_{0}\right) \pm i \sin \left(4 \theta_{0}\right)$, and a particular solution of (92) is given by

$$
\left\{\begin{array}{l}
a(n)=-S_{3}\left\{(2 n-1) \sin \left(2 \theta_{0}\right)+\sin \left(2(n+1) \theta_{0}\right)+\sin \left(2 n \theta_{0}\right)\right\}, \\
b(n)=2 S_{3}\left\{n\left[\sin \left(\theta_{0}\right)+\sin \left(3 \theta_{0}\right)\right]+\sin \left((2 n+1) \theta_{0}\right)+\sin \left((2 n-1) \theta_{0}\right)\right\}, \\
c(n)=-S_{3}\left\{(2 n+1) \sin \left(2 \theta_{0}\right)+\sin \left(2 n \theta_{0}\right)+\sin \left(2(n-1) \theta_{0}\right)\right\}, \\
d(n)=-S_{4}\left\{(2 n+1) \sin \left(2 \theta_{0}\right)-\sin \left(2(2 n+1) \theta_{0}\right)\right\}, \\
e(n)=4 S_{4}\left\{n\left[\sin \left(\theta_{0}\right)+\sin \left(3 \theta_{0}\right)\right]+\sin \left(\theta_{0}\right)-\sin \left((4 n+1) \theta_{0}\right)\right\}, \\
f(n)=-2 S_{4}\left\{n\left[4 \sin \left(2 \theta_{0}\right)+\sin \left(4 \theta_{0}\right)\right]-3 \sin \left(4 n \theta_{0}\right)\right\}, \\
g(n)=4 S_{4}\left\{n\left[\sin \left(\theta_{0}\right)+\sin \left(3 \theta_{0}\right)\right]-\sin \left(\theta_{0}\right)-\sin \left((4 n-1) \theta_{0}\right)\right\}, \\
h(n)=-S_{4}\left\{(2 n-1) \sin \left(2 \theta_{0}\right)-\sin \left(2(2 n-1) \theta_{0}\right)\right\},
\end{array}\right.
$$

where $S_{3}=\frac{1}{\sin \left(2 \theta_{0}\right)}$, and $S_{4}=\frac{1}{\sin \left(4 \theta_{0}\right)-2 \sin \left(2 \theta_{0}\right)}$. From (89)-(91), we have

$$
\begin{aligned}
Z_{1}\left(x_{n}, y_{n}, n\right)= & A_{1}\left(x_{n}^{2}-2 x_{n} y_{n} \cos \left(\theta_{0}\right)+y_{n}^{2}, n+\frac{1}{\theta_{0}} \arctan \left(\frac{y_{n} \sin \left(\theta_{0}\right)}{x_{n}-y_{n} \cos \left(\theta_{0}\right)}\right)\right) \\
& +a(n) x_{n}^{2}+b(n) x_{n} y_{n}+c(n) y_{n}^{2}+d(n) x_{n}^{4}+e(n) x_{n}^{3} y_{n}+f(n) x_{n}^{2} y_{n}^{2}+g(n) x_{n} y_{n}^{3}+h(n) y_{n}^{4} .
\end{aligned}
$$

We will now choose $Z_{1}$ as simple as possible, that is, $A_{1} \equiv 0$ in (94), and so

$$
\begin{aligned}
Z_{1}\left(x_{n}, y_{n}, n\right)= & a(n) x_{n}^{2}+b(n) x_{n} y_{n}+c(n) y_{n}^{2}+d(n) x_{n}^{4} \\
& +e(n) x_{n}^{3} y_{n}+f(n) x_{n}^{2} y_{n}^{2} \\
& +g(n) x_{n} y_{n}^{3}+h(n) y_{n}^{4}
\end{aligned}
$$

where $a(n), b(n), \ldots$, and $h(n)$ are given by (93). Just like in case 1 in the example of Sect. 4, an approximation $I_{A}\left(x_{n}, y_{n}, n\right)$ of an invariant $I\left(x_{n}, y_{n}, n\right)=$ constant for (81) is constructed, and is given by

$$
\begin{aligned}
I_{A}\left(x_{n}, y_{n}, n\right)= & x_{n}^{2}-2 x_{n} y_{n} \cos \left(\theta_{0}\right)+y_{n}^{2} \\
& +\varepsilon\left\{a(n) x_{n}^{2}+b(n) x_{n} y_{n}+c(n) y_{n}^{2}\right. \\
& +d(n) x_{n}^{4}+e(n) x_{n}^{3} y_{n}+f(n) x_{n}^{2} y_{n}^{2} \\
& \left.+g(n) x_{n} y_{n}^{3}+h(n) y_{n}^{4}\right\},
\end{aligned}
$$

or equivalently,

$$
\begin{aligned}
I_{A}\left(x_{n+1}, y_{n+1}, n+1\right)= & I_{A}\left(x_{n}, y_{n}, n\right) \\
& +\varepsilon^{2} R\left(x_{n}, y_{n}, n ; \varepsilon\right),
\end{aligned}
$$

where

$$
\begin{aligned}
\varepsilon^{2} R & \left(x_{n}, y_{n}, n ; \varepsilon\right) \\
= & -2 f_{1}\left(f_{2}-f_{2,0}-\varepsilon f_{2,1}\right) \cos \left(\theta_{0}\right)+\left(f_{2}^{2}-f_{2,0}^{2}\right. \\
& \left.-2 \varepsilon f_{2,0} f_{2,1}\right)+\varepsilon\left\{b(n+1) f_{1}\left(f_{2}-f_{2,0}\right)\right. \\
& +c(n+1)\left(f_{2}^{2}-f_{2,0}^{2}\right)+e(n+1) f_{1}^{3}\left(f_{2}-f_{2,0}\right) \\
& +f(n+1) f_{1}^{2}\left(f_{2}^{2}-f_{2,0}^{2}\right) \\
& +g(n+1) f_{1}\left(f_{2}^{3}-f_{2,0}^{3}\right) \\
& \left.+h(n+1)\left(f_{2}^{4}-f_{2,0}^{4}\right)\right\},
\end{aligned}
$$


where $f_{1}, f_{2}, f_{2,0}$ and $f_{2,1}$ are given by (15) and (81). Since the Van der Pol system (81) is $n$-independent, and $b(n), c(n), e(n), f(n), g(n)$, and $h(n)$ are of $O(n)$, then $R$ is unbounded in $n$ and of $O(n)$. From (97), it follows that

$$
\begin{aligned}
I_{A}\left(x_{n}, y_{n}, n\right)= & I_{A}\left(x_{0}, y_{0}, 0\right) \\
& +\varepsilon^{2} \sum_{i=0}^{n-1} R\left(x_{i}, y_{i}, i ; \varepsilon\right) .
\end{aligned}
$$

From (97) and (99), it can be shown that

$$
\begin{aligned}
I_{A}\left(x_{n}, y_{n}, n\right)= & x_{0}^{2}-2 x_{0} y_{0} \cos \left(\theta_{0}\right)+y_{0}^{2} \\
& +O\left(\varepsilon^{2} n^{2}\right),
\end{aligned}
$$

it then follows that

$$
\left\{\begin{array}{l}
I_{A}\left(x_{n}, y_{n}, n\right)=\text { constant }+O\left(\varepsilon^{2}\right) \\
\quad \text { for } n=O(1), \\
I_{A}\left(x_{n}, y_{n}, n\right)=\text { constant }+O(\varepsilon) \\
\quad \text { for } n=O\left(\frac{1}{\sqrt{\varepsilon}}\right) .
\end{array}\right.
$$

So far, only one approximation of a first integral has been determined. Another (functionally independent) approximation of a first integral can also be obtained in a similar and straightforward way as follows from the next subsection.

\subsection{Case 2}

In this case, we take

$$
\left\{\begin{array}{l}
A_{0} \equiv n+\frac{1}{\theta_{0}} \arctan \left(\frac{y_{n} \sin \left(\theta_{0}\right)}{x_{n}-y_{n} \cos \left(\theta_{0}\right)}\right) \\
\mu_{1,0}\left(x_{n}, y_{n}, n\right)=0
\end{array}\right.
$$

So, from (83) and (86), we find

$$
\mu_{2,0}=\frac{1}{y_{n}}\left(n+\frac{1}{\theta_{0}} \arctan \left(\frac{y_{n} \sin \left(\theta_{0}\right)}{x_{n}-y_{n} \cos \left(\theta_{0}\right)}\right)\right) .
$$

By substituting the values of $\mu_{1,0}$ and $\mu_{2,0}$ into (20), the $O(\varepsilon)$-problem then becomes

$$
\begin{aligned}
& Z_{1}\left(x_{n}, y_{n}, n\right) \\
& \quad=Z_{1}\left(y_{n}, 2 y_{n} \cos \left(\theta_{0}\right)-x_{n}, n+1\right) \\
& \quad+\frac{2 y_{n}\left(x_{n}-y_{n} \cos \left(\theta_{0}\right)\right) \sin \left(\theta_{0}\right)\left(y_{n}^{2}-1\right)}{\theta_{0}\left(x_{n}^{2}-2 x_{n} y_{n} \cos \left(\theta_{0}\right)+y_{n}^{2}\right)},
\end{aligned}
$$

where $Z_{1}\left(x_{n}, y_{n}, n\right)=\mu_{1,1}\left(x_{n}, y_{n}, n\right) x_{n}+\mu_{2,1}\left(x_{n}\right.$, $\left.y_{n}, n\right) y_{n}$. When we put $\mu_{2,0}=0$ instead of $\mu_{1,0}=0$, then $\mu_{1,0}=\frac{1}{x_{n}}\left(n+\frac{1}{\theta_{0}} \arctan \left(\frac{y_{n} \sin \left(\theta_{0}\right)}{x_{n}-y_{n} \cos \left(\theta_{0}\right)}\right)\right)$. Again the so-obtained $Z_{1}$ function has to satisfy (104). According to (84) and (85), the general solution $Z_{1, h}$ of the homogeneous equation related to (104) is

$$
\begin{aligned}
& Z_{1, h}\left(x_{n}, y_{n}, n\right) \\
& =A_{1}\left(x_{n}^{2}-2 x_{n} y_{n} \cos \left(\theta_{0}\right)+y_{n}^{2},\right. \\
& \left.\quad n+\frac{1}{\theta_{0}} \arctan \left(\frac{y_{n} \sin \left(\theta_{0}\right)}{x_{n}-y_{n} \cos \left(\theta_{0}\right)}\right)\right) .
\end{aligned}
$$

Now, we are going to construct a particular solution of (104). Since $\frac{2 \sin \left(\theta_{0}\right)}{\theta_{0}\left(x_{n}^{2}-2 x_{n} y_{n} \cos \left(\theta_{0}\right)+y_{n}^{2}\right)}$ satisfies the homogeneous equation related to (104), we look for a particular solution in the form

$$
\begin{aligned}
Z_{1, p} & \left(x_{n}, y_{n}, n\right) \\
= & \frac{2 \sin \left(\theta_{0}\right)}{\theta_{0}\left(x_{n}^{2}-2 x_{n} y_{n} \cos \left(\theta_{0}\right)+y_{n}^{2}\right)} \\
& \times\left\{a(n) x_{n}^{2}+b(n) x_{n} y_{n}+c(n) y_{n}^{2}+d(n) x_{n}^{4}\right. \\
& \left.+e(n) x_{n}^{3} y_{n}+f(n) x_{n}^{2} y_{n}^{2}+g(n) x_{n} y_{n}^{3}+h(n) y_{n}^{4}\right\} .
\end{aligned}
$$

By substituting (106) into (104), and by taking apart in the resulting equation the coefficients of $x_{n}^{2}, x_{n} y_{n}$, $y_{n}^{2}, x_{n}^{4}, x_{n}^{3} y_{n}, x_{n}^{2} y_{n}^{2}, x_{n} y_{n}^{3}$, and $y_{n}^{4}$, and setting these coefficients equal to zero, we will have the following system: 


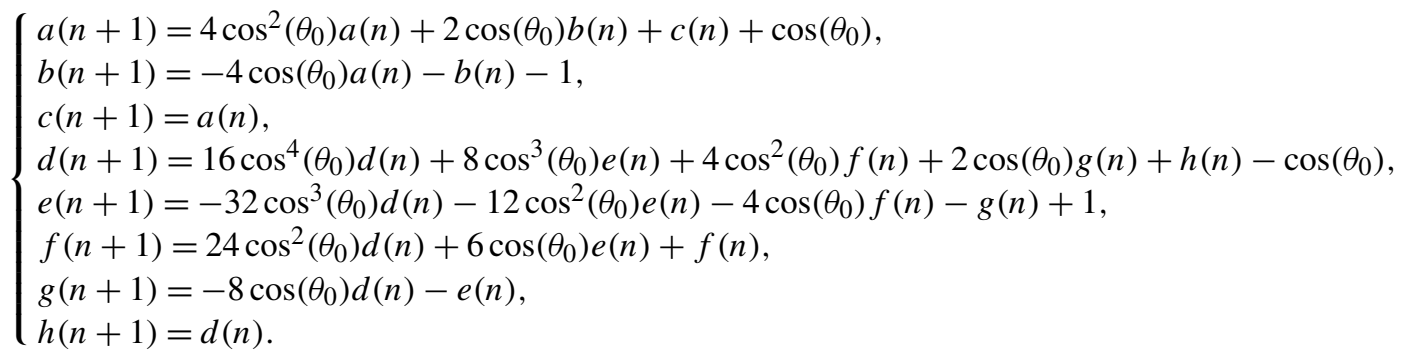

The eigenvalues of the homogeneous system related to (107) are $1,1, \cos \left(2 \theta_{0}\right) \pm i \sin \left(2 \theta_{0}\right), \cos \left(2 \theta_{0}\right) \pm i \sin \left(2 \theta_{0}\right)$, and $\cos \left(4 \theta_{0}\right) \pm i \sin \left(4 \theta_{0}\right)$, and a particular solution of (107) is given by

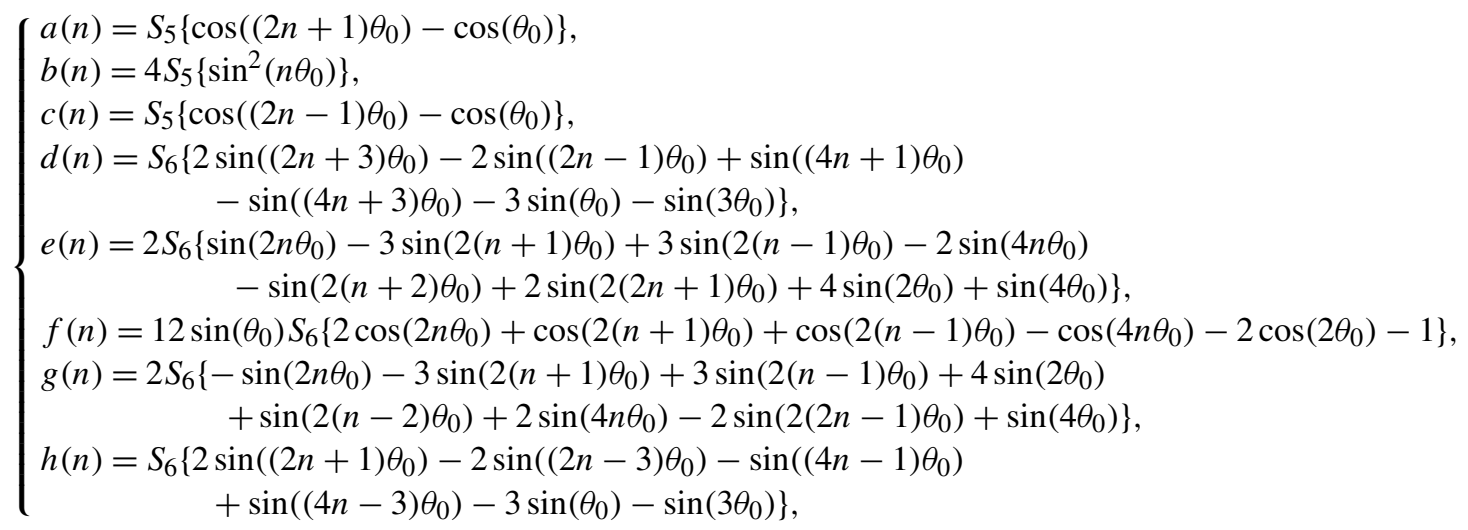

where $S_{5}=-\frac{1}{4 \sin ^{2}\left(\theta_{0}\right)}$, and $S_{6}=\frac{1}{64 \sin ^{5}\left(\theta_{0}\right) \cos \left(\theta_{0}\right)}$. From (104)-(106), we have

$$
\begin{aligned}
Z_{1}\left(x_{n}, y_{n}, n\right)= & A_{1}\left(x_{n}^{2}-2 x_{n} y_{n} \cos \left(\theta_{0}\right)+y_{n}^{2}, n+\frac{1}{\theta_{0}} \arctan \left(\frac{y_{n} \sin \left(\theta_{0}\right)}{x_{n}-y_{n} \cos \left(\theta_{0}\right)}\right)\right) \\
& +\frac{2 \sin \left(\theta_{0}\right)}{\theta_{0}\left(x_{n}^{2}-2 x_{n} y_{n} \cos \left(\theta_{0}\right)+y_{n}^{2}\right)}\left\{a(n) x_{n}^{2}+b(n) x_{n} y_{n}+c(n) y_{n}^{2}\right. \\
& \left.+d(n) x_{n}^{4}+e(n) x_{n}^{3} y_{n}+f(n) x_{n}^{2} y_{n}^{2}+g(n) x_{n} y_{n}^{3}+h(n) y_{n}^{4}\right\} .
\end{aligned}
$$

We will now choose $Z_{1}$ as simple as possible, that is, $A_{1} \equiv 0$ in (109), and so

$$
\begin{aligned}
Z_{1}\left(x_{n}, y_{n}, n\right)= & \frac{2 \sin \left(\theta_{0}\right)}{\theta_{0}\left(x_{n}^{2}-2 x_{n} y_{n} \cos \left(\theta_{0}\right)+y_{n}^{2}\right)}\left\{a(n) x_{n}^{2}+b(n) x_{n} y_{n}+c(n) y_{n}^{2}+d(n) x_{n}^{4}\right. \\
& \left.+e(n) x_{n}^{3} y_{n}+f(n) x_{n}^{2} y_{n}^{2}+g(n) x_{n} y_{n}^{3}+h(n) y_{n}^{4}\right\},
\end{aligned}
$$

where $a(n), b(n), \ldots$, and $h(n)$ are given by (108). Just like in case 2 in Sect. 4 of this paper, an approximation $I_{A}\left(x_{n}, y_{n}, n\right)$ of an invariant $I\left(x_{n}, y_{n}, n\right)=$ constant for (81) is constructed, and is given by

$$
\begin{aligned}
I_{A}\left(x_{n}, y_{n}, n\right)= & n+\frac{1}{\theta_{0}} \arctan \left(\frac{y_{n} \sin \left(\theta_{0}\right)}{x_{n}-y_{n} \cos \left(\theta_{0}\right)}\right)+\frac{2 \varepsilon \sin \left(\theta_{0}\right)}{\theta_{0}\left(x_{n}^{2}-2 x_{n} y_{n} \cos \left(\theta_{0}\right)+y_{n}^{2}\right)}\left\{a(n) x_{n}^{2}\right. \\
& \left.+b(n) x_{n} y_{n}+c(n) y_{n}^{2}+d(n) x_{n}^{4}+e(n) x_{n}^{3} y_{n}+f(n) x_{n}^{2} y_{n}^{2}+g(n) x_{n} y_{n}^{3}+h(n) y_{n}^{4}\right\},
\end{aligned}
$$

and,

$$
I_{A}\left(x_{n+1}, y_{n+1}, n+1\right)=I_{A}\left(x_{n}, y_{n}, n\right)+\varepsilon^{2} R\left(x_{n}, y_{n}, n ; \varepsilon\right),
$$


where,

$$
\begin{aligned}
\varepsilon^{2} R\left(x_{n}, y_{n}, n ; \varepsilon\right)= & \frac{1}{\theta_{0}}\left\{\arctan \left(\frac{f_{2} \sin \left(\theta_{0}\right)}{f_{1}-f_{2} \cos \left(\theta_{0}\right)}\right)-\arctan \left(\frac{f_{2,0} \sin \left(\theta_{0}\right)}{f_{1}-f_{2,0} \cos \left(\theta_{0}\right)}\right)-\frac{f_{1} f_{2,1} \sin \left(\theta_{0}\right)}{f_{1}^{2}-2 f_{1} f_{2,0} \cos \left(\theta_{0}\right)+f_{2,0}^{2}} \varepsilon\right\} \\
& +\frac{2 \varepsilon \sin \left(\theta_{0}\right)}{\theta_{0}}\left\{\frac { 1 } { f _ { 1 } ^ { 2 } - 2 f _ { 1 } f _ { 2 } \operatorname { c o s } ( \theta _ { 0 } ) + f _ { 2 } ^ { 2 } } \left[a(n+1) f_{1}^{2}+b(n+1) f_{1} f_{2}+c(n+1) f_{2}^{2}\right.\right. \\
& \left.+d(n+1) f_{1}^{4}+e(n+1) f_{1}^{3} f_{2}+f(n+1) f_{1}^{2} f_{2}^{2}+g(n+1) f_{1} f_{2}^{3}+h(n+1) f_{2}^{4}\right] \\
& -\frac{1}{f_{1}^{2}-2 f_{1} f_{2,0} \cos \left(\theta_{0}\right)+f_{2,0}^{2}}\left[a(n+1) f_{1}^{2}+b(n+1) f_{1} f_{2,0}+c(n+1) f_{2,0}^{2}+d(n+1) f_{1}^{4}\right. \\
& \left.\left.+e(n+1) f_{1}^{3} f_{2,0}+f(n+1) f_{1}^{2} f_{2,0}^{2}+g(n+1) f_{1} f_{2,0}^{3}+h(n+1) f_{2,0}^{4}\right]\right\},
\end{aligned}
$$

where $f_{1}, f_{2}, f_{2,0}$ and $f_{2,1}$ are given by (15) and (81). Since the Van der Pol system (81) is $n$-independent, and $a(n), b(n), c(n), d(n), e(n), f(n), g(n)$, and $h(n)$ are bounded, then $R$ is bounded. From (112), it follows that

$$
\begin{aligned}
I_{A}\left(x_{n}, y_{n}, n\right)= & I_{A}\left(x_{0}, y_{0}, 0\right) \\
& +\varepsilon^{2} \sum_{i=0}^{n-1} R\left(x_{i}, y_{i}, i ; \varepsilon\right),
\end{aligned}
$$

where $I_{A}\left(x_{0}, y_{0}, 0\right)=\frac{1}{\theta_{0}} \arctan \left(\frac{y_{0} \sin \left(\theta_{0}\right)}{x_{0}-y_{0} \cos \left(\theta_{0}\right)}\right)=$ constant. From (112) and (114), it can be shown that

$I_{A}\left(x_{n}, y_{n}, n\right)=$ constant $+O\left(\varepsilon^{2} n\right)$,

it then follows that

$$
\left\{\begin{array}{l}
I_{A}\left(x_{n}, y_{n}, n\right)=\text { constant }+O\left(\varepsilon^{2}\right) \\
\quad \text { for } n=O(1), \\
I_{A}\left(x_{n}, y_{n}, n\right)=\text { constant }+O(\varepsilon) \\
\quad \text { for } n=O\left(\frac{1}{\varepsilon}\right) .
\end{array}\right.
$$

To see how the solution of the Van der Pol difference system (81) behaves, it is more convenient to work in polar coordinates

$$
\left\{\begin{array}{l}
x_{n}=r(n) \sin \left(\varphi_{n}+\theta_{0}\right) \\
y_{n}=r(n) \sin \left(\varphi_{n}\right)
\end{array}\right.
$$

where $r(n)$ is approximated by $r_{0}(n)+\varepsilon r_{1}(n)+$ $O\left(\varepsilon^{2}\right)$. Without loss of generality, it can be assumed that the first integral as given by (96) can be written as $I_{A}\left(x_{n}, y_{n}, n\right)=c_{0}+\varepsilon c_{1}+O\left(\varepsilon^{2}\right)$, where $c_{0}, c_{1}, \ldots$ are constants. By substituting (117) into (96), by using $r(n)=r_{0}(n)+\varepsilon r_{1}(n)+O\left(\varepsilon^{2}\right)$, and by collecting terms of equal powers in $\varepsilon$, we then obtain $r_{0}(n), r_{1}(n), \ldots$ after some elementary calculations, yielding

$$
\left\{\begin{array}{l}
c_{0}=r_{0}^{2}(n) \sin ^{2}\left(\theta_{0}\right), \\
r_{1}(n)=\frac{c_{1}}{2 r_{0}(n) \sin ^{2}\left(\theta_{0}\right)}-\frac{n r_{0}(n)}{4}\left(r_{0}^{2}(n)-4\right) \\
\quad+\text { bounded terms } \text { in } n .
\end{array}\right.
$$

From (118), it follows that $r(n) \rightarrow 2$ for increasing $n$. This result is in agreement with the results for the solution of the Van der Pol differential system.

\section{Conclusions and remarks}

In this paper, the concept of invariance factors for system of first order difference equations has been presented, and proofs to justify the obtained results have been given. It has been shown that all invariance factors have to satisfy a functional equation. The presented perturbation method based on invariance factors has been applied successfully to weakly perturbed, linear, and nonlinear oscillator equations. Since we used rather naive approximations for the invariance factors, it turned out that not all $O(\varepsilon)$ approximations of first integrals are valid on iteration scales of order $\frac{1}{\varepsilon}$ (see, for instance, (48) and (53)). In fact, the terms $(2 n-1) x_{n}^{2},-4 n \cos \left(\theta_{0}\right) x_{n} y_{n}$, and $(2 n+1) y_{n}^{2}$ in (48) cause secular behavior in $n$. This secular behavior can be avoided by introducing multiple scales in the invariance factors. The results for the Van der Pol system (81) without these secular terms will be presented in a forthcoming paper, and these results are valid on much larger iteration scales. The goal 
of this paper is to present the perturbation method, and the method is applied to (relatively) simple problems. In another forthcoming paper a weakly perturbed, but essentially nonlinear problem will be studied (that is, when $\varepsilon=0$ the problem is nonlinear). Showing that the method can be applied to a large class of problems.

Open Access This article is distributed under the terms of the Creative Commons Attribution Noncommercial License which permits any noncommercial use, distribution, and reproduction in any medium, provided the original author(s) and source are credited.

\section{Appendix}

To obtain the general solution of the functional equation,

$Z_{0}\left(x_{n}, y_{n}, n\right)=Z_{0}\left(y_{n}, 2 y_{n} \cos \left(\theta_{0}\right)-x_{n}, n+1\right)$,

we apply the method of separation of variables by substituting $K\left(x_{n}, y_{n}\right) N(n)$ for $Z_{0}\left(x_{n}, y_{n}, n\right)$ into (119), yielding

$K\left(x_{n}, y_{n}\right) N(n)=K\left(y_{n}, 2 y_{n} \cos \left(\theta_{0}\right)-x_{n}\right) N(n+1)$.

So,

$\frac{K\left(x_{n}, y_{n}\right)}{K\left(y_{n}, 2 y_{n} \cos \left(\theta_{0}\right)-x_{n}\right)}=\frac{N(n+1)}{N(n)}=\gamma$,

where $\gamma$ is a (complex-valued) separation constant. From (121), it follows that

$K\left(x_{n}, y_{n}\right)=\gamma K\left(y_{n}, 2 y_{n} \cos \left(\theta_{0}\right)-x_{n}\right), \quad$ and

$N(n+1)=\gamma N(n)$.

Now the transformation $K\left(x_{n}, y_{n}\right)=G(z, w)$, where $z=a x_{n}+b y_{n}$, and $w=c x_{n}+d y_{n}$ in which $a, b, c$, and $d$ are (complex-valued) constants, will be used to obtain

$$
\begin{aligned}
G(z, w)= & \gamma G\left(a y_{n}+b\left(2 y_{n} \cos \left(\theta_{0}\right)-x_{n}\right), c y_{n}\right. \\
& \left.+d\left(2 y_{n} \cos \left(\theta_{0}\right)-x_{n}\right)\right),
\end{aligned}
$$

or equivalently,

$$
\begin{aligned}
G(z, w)= & \gamma G\left(-b x_{n}+\left(a+2 b \cos \left(\theta_{0}\right)\right) y_{n},\right. \\
& \left.-d x_{n}+\left(c+2 d \cos \left(\theta_{0}\right)\right) y_{n}\right) .
\end{aligned}
$$

Now, $a, b, c, d$ will be chosen such that (124) becomes

$G(z, w)=\gamma G\left(\lambda_{1} z, \lambda_{2} w\right)$,

where $\lambda_{1}$ and $\lambda_{2}$ are (complex-valued) constants. From (124) and (125), it then follows that

$$
\left\{\begin{array}{l}
\lambda_{1} a+b=0, \\
a+\left(2 \cos \left(\theta_{0}\right)-\lambda_{1}\right) b=0, \\
\lambda_{2} c+d=0, \\
c+\left(2 \cos \left(\theta_{0}\right)-\lambda_{2}\right) d=0 .
\end{array}\right.
$$

To have a nontrivial solution for $a, b, c$, and $d$, it follows that

$\lambda_{1,2}=\exp \left( \pm i \theta_{0}\right)$

We observe that $\lambda_{1,2}$ are the eigenvalues of the matrix corresponding to the system of linear, ordinary difference equations (28) with $\varepsilon=0$. Now by applying the method of separation of variables to (125), we substitute $H(z) F(w)$ for $G(z, w)$ into (125), and we obtain

$\frac{H(z)}{H\left(\exp \left(i \theta_{0}\right) z\right)}=\frac{\gamma F\left(\exp \left(-i \theta_{0}\right) w\right)}{F(w)}=\alpha$,

where $\alpha$ is a (complex-valued) separation constant. So, we now have two functional equations with complex variables $z$ and $w$. First, we solve

$H(z)=\alpha H\left(\exp \left(i \theta_{0}\right) z\right)$

and then by a simple transformation we obtain the solution of the equation for $F$. To obtain the general solution of (129), we introduce

$$
\begin{gathered}
\alpha=\alpha_{1}+i \alpha_{2}, \quad z=r \exp (i \theta), \quad H(z)=J(r, \theta), \\
\text { and } \quad J(r, \theta)=J_{1}(r, \theta)+i J_{2}(r, \theta),
\end{gathered}
$$

where $\alpha_{1}$ and $\alpha_{2}$ are real numbers, and where $J_{1}$ and $J_{2}$ are real valued functions. Then, (129) becomes

$$
\begin{aligned}
J_{1}(r, \theta)+i J_{2}(r, \theta)= & \left(\alpha_{1}+i \alpha_{2}\right)\left(J_{1}\left(r, \theta+\theta_{0}\right)\right. \\
& \left.+i J_{2}\left(r, \theta+\theta_{0}\right)\right)
\end{aligned}
$$

and so, we get two equations from (131)

$$
J_{1}(r, \theta)=\alpha_{1} J_{1}\left(r, \theta+\theta_{0}\right)-\alpha_{2} J_{2}\left(r, \theta+\theta_{0}\right),
$$

and,

$$
J_{2}(r, \theta)=\alpha_{2} J_{1}\left(r, \theta+\theta_{0}\right)+\alpha_{1} J_{2}\left(r, \theta+\theta_{0}\right) .
$$


First, we consider the case $\alpha_{2}=0$. Then (132) and (133) become

$J_{1}(r, \theta)=\alpha_{1} J_{1}\left(r, \theta+\theta_{0}\right)$,

and

$J_{2}(r, \theta)=\alpha_{1} J_{2}\left(r, \theta+\theta_{0}\right)$.

The general solutions of (134) and (135) can readily be obtained, and are given by

$J_{1}(r, \theta)=\alpha_{1}^{-\frac{\theta}{\theta_{0}}} j_{1}(r), \quad$ and $\quad J_{2}(r, \theta)=\alpha_{1}^{-\frac{\theta}{\theta_{0}}} j_{2}(r)$,

where $j_{1}(r)$ and $j_{2}(r)$ are arbitrary functions. Now from (130), we obtain

$H(z)=\alpha^{\frac{i}{\theta_{0}} \ln \left(\frac{z}{|z|}\right)} h(|z|)$,

where $h$ is a complex valued function. Now from (128), we have

$F(w)=\frac{\gamma}{\alpha} F\left(\exp \left(-i \theta_{0}\right) w\right)$,

which can be solved by some simple transformations

$\alpha \rightarrow \frac{\gamma}{\alpha}, \quad \theta_{0} \rightarrow-\theta_{0}, \quad z \rightarrow w, \quad$ and $\quad H \rightarrow F$,

therefore, we have

$F(w)=\left(\frac{\gamma}{\alpha}\right)^{-\frac{i}{\theta_{0}} \ln \left(\frac{w}{|w|}\right)} f(|w|)$.

Observing that $G(z, w)=H(z) F(w)$, we obtain

$G(z, w)=\gamma^{-\frac{i}{\theta_{0}} \ln \left(\frac{w}{|w|}\right)} g\left(|z|,|w|, \frac{z w}{|z w|}\right)$.

By taking $a=c=1$ in (126), it follows that

$z=r \exp (i \theta)=x_{n}-y_{n} \lambda_{1}$,

and

$w=\tilde{r} \exp (i \widetilde{\theta})=x_{n}-y_{n} \lambda_{2}$,

therefore,

$r=\widetilde{r}=\sqrt{x_{n}^{2}-2 x_{n} y_{n} \cos \left(\theta_{0}\right)+y_{n}^{2}}$, and

$\theta=-\widetilde{\theta}=\arctan \left(\frac{-y_{n} \sin \left(\theta_{0}\right)}{x_{n}-y_{n} \cos \left(\theta_{0}\right)}\right)$.

So, $G(z, w)$ will be given by

$G(z, w)=\gamma^{-\frac{i}{\theta_{0}} \ln \left(\frac{w}{|w|}\right)} g(|z|)$.

From (122), we have

$N(n)=\gamma^{n}$,

and since $Z_{0}\left(x_{n}, y_{n}, n\right)=K\left(x_{n}, y_{n}\right) N(n)$, and $K\left(x_{n}, y_{n}\right)=G(z, w)$, the general solution for $Z_{0}\left(x_{n}\right.$, $\left.y_{n}, n\right)$ will be given by

$$
\begin{aligned}
Z_{0}\left(x_{n}, y_{n}, n\right)= & A_{0}\left(x_{n}^{2}-2 x_{n} y_{n} \cos \left(\theta_{0}\right)+y_{n}^{2},\right. \\
& \left.n+\frac{1}{\theta_{0}} \arctan \left(\frac{y_{n} \sin \left(\theta_{0}\right)}{x_{n}-y_{n} \cos \left(\theta_{0}\right)}\right)\right) .
\end{aligned}
$$

If we consider $\alpha_{2} \neq 0$, we will have the same results for the final solution $Z_{0}\left(x_{n}, y_{n}, n\right)$ (for the full analysis of this problem we refer the reader to [15]). To check the correctness of our results, we have to show that $\Delta Z_{0}\left(x_{n}, y_{n}, n\right)=0$ (see (31)). By some elementary calculations, we see that $\Delta\left(x_{n}^{2}-2 x_{n} y_{n} \cos \left(\theta_{0}\right)+\right.$ $\left.y_{n}^{2}\right)=0$. And for the second argument of $A_{0}$ in (148), we have to prove that $\Delta\left(n+\frac{1}{\theta_{0}} \arctan \left(\frac{y_{n} \sin \left(\theta_{0}\right)}{x_{n}-y_{n} \cos \left(\theta_{0}\right)}\right)\right)=$ 0 , and this means that

$$
\begin{aligned}
n+ & \frac{1}{\theta_{0}} \arctan \left(\frac{y_{n} \sin \left(\theta_{0}\right)}{x_{n}-y_{n} \cos \left(\theta_{0}\right)}\right)=n+1 \\
& +\frac{1}{\theta_{0}} \arctan \left(\frac{\left(2 y \cos \left(\theta_{0}\right)-x\right) \sin \left(\theta_{0}\right)}{y_{n}-\left(2 y \cos \left(\theta_{0}\right)-x\right) \cos \left(\theta_{0}\right)}\right) .
\end{aligned}
$$

To do this, we have to verify that if $\tan (\xi)=$ $\frac{y_{n} \sin \left(\theta_{0}\right)}{x_{n}-y_{n} \cos \left(\theta_{0}\right)}$, then $\tan \left(\xi-\theta_{0}\right)=\frac{\left(2 y \cos \left(\theta_{0}\right)-x\right) \sin \left(\theta_{0}\right)}{y_{n}-\left(2 y \cos \left(\theta_{0}\right)-x\right) \cos \left(\theta_{0}\right)}$, where $\xi$ is an arbitrary constant, and this can be readily checked by simple substitutions.

\section{References}

1. Van Horssen, W.T.: On asymptotic approximations of first integrals for differential and difference equations. J. Indones. Math. Soc. 13, 1-15 (2007) 
2. Van Horssen, W.T.: On integrating factors for ordinary differential equations. Nieuw Arch. Wisk. 15, 15-26 (1997)

3. Van Horssen, W.T.: A perturbation based on integrating factors. SIAM J. Appl. Math 59, 1427-1443 (1999)

4. Van Horssen, W.T.: A perturbation based on integrating vectors and multiple scales. SIAM J. Appl. Math 59, 1444 1467 (1999)

5. Agarwal, R.P.: Difference Equations and Inequalities. Dekker, New York (1992)

6. Elaydi, S.N.: An Introduction to Difference Equations. Springer, New York (1996)

7. Van Horssen, W.T.: On invariance factors and invariance vectors for difference equations. J. Differ. Equ. Apl. 8, 1133-1146 (2002)

8. Kuczma, M.: Functional Equations in a Single Variable. Polish Scientific, Warsaw (1968)

9. Kuczma, M., Choczewski, B., Ger, R.: Iterative Functional Equations. Cambridge University Press, New York (1990)

10. Aczel, J., Dhombres, J.: Functional Equations in Several Variables. Cambridge University Press, New York (1989)
11. Risteski, I., Covachev, V.: Complex Vector Functional Equations. World Scientific, Singapore (2001)

12. Bowman, D.: q-difference operators, orthogonal polynomials, and symmetric expansions. Am. Math. Soc. 159(757) (2002)

13. Small, C.G.: Functional Equations and How to Solve Them. Springer, Berlin (2007)

14. Van Horssen, W.T., ter Brake, M.C.: On the multiple scales perturbation method for difference equations. Nonlinear Dyn. 55, 401-418 (2009)

15. Rafei, M., Van Horssen, W.T.: On constructing solutions for the functional equation $Z(x, y, n)=Z\left(a_{11} x+\right.$ $\left.a_{12} y, a_{21} x+a_{22} y, n+1\right)$. J. Math. Anal. Appl. (2009) (submitted)

16. Mickens, R.E.: Oscillations in Planar Dynamic Systems. World Scientific, River Edge (1996)

17. Nayfeh, A.H.: Perturbation Methods. Wiley, New York (1973)

18. Potts, R.B.: Van der Pol difference equation. Nonlinear Anal. 7, 801-812 (1983) 